\title{
Laser-assisted decay spectroscopy for the ground states of ${ }^{180,182} \mathrm{Au}$
}

R. D. Harding $\odot,{ }^{1,2}$ A. N. Andreyev $\odot,{ }^{1,3, *}$ A. E. Barzakh, ${ }^{4}$ D. Atanasov, ${ }^{5, \dagger}$ J. G. Cubiss, ${ }^{1}$ P. Van Duppen, ${ }^{6}$ M. Al Monthery, ${ }^{1}$ N. A. Althubiti, ${ }^{7,8}$ B. Andel, ${ }^{6,9}$ S. Antalic, ${ }^{9}$ K. Blaum, ${ }^{5}$ T. E. Cocolios, ${ }^{6,8}$ T. Day Goodacre, $, 2,8, \$$ A. de Roubin,,${ }^{5,8}$

G. J. Farooq-Smith ${ }^{6,8}$ D. V. Fedorov, ${ }^{4}$ V. N. Fedosseev, ${ }^{2}$ D. A. Fink,,${ }^{2,5}$ L. P. Gaffney, ${ }^{2,10}$ L. Ghys, ${ }^{6, \|}$ D. T. Joss, ${ }^{10}$ F. Herfurth, ${ }^{11}$ M. Huyse, ${ }^{6}$ N. Imai, ${ }^{12}$ S. Kreim, ${ }^{2,5}$ D. Lunney, ${ }^{13, \pi l}$ K. M. Lynch, ${ }^{2,8}$ V. Manea, ${ }^{5, \pi}$ B. A. Marsh, ${ }^{2}$ Y. Martinez Palenzuela,,${ }^{2,6}$

P. L. Molkanov, ${ }^{4}$ D. Neidherr, ${ }^{11}$ R. D. Page, ${ }^{10}$ A. Pastore, ${ }^{1}$ M. Rosenbusch, ${ }^{14}$ R. E. Rossel,,${ }^{2,15}$ S. Rothe, ${ }^{2,15}$

L. Schweikhard, ${ }^{14}$ M. D. Seliverstov, ${ }^{4}$ S. Sels, ${ }^{6}$ C. Van Beveren, ${ }^{6}$ E. Verstraelen, ${ }^{6}$ A. Welker, ${ }^{2,16}$

F. Wienholtz, ${ }^{2,17,14}$ R. N. Wolf, ${ }^{5,14, \#}$ and K. Zuber ${ }^{16}$

${ }^{1}$ Department of Physics, University of York, York, YO10 5DD, United Kingdom

${ }^{2}$ CERN, 1211, Geneva 23, Switzerland

${ }^{3}$ Advanced Science Research Center (ASRC), Japan Atomic Energy Agency, Tokai-mura, Japan

${ }^{4}$ Petersburg Nuclear Physics Institute, NRC Kurchatov Institute, 188300 Gatchina, Russia

${ }^{5}$ Max-Planck-Institut für Kernphysik, Saupfercheckweg 1, 69117 Heidelberg, Germany

${ }^{6}$ KU Leuven, Instituut voor Kern- en Stralingsfysica, 3001 Leuven, Belgium

${ }^{7}$ Physics Department, Faculty of Science, Jouf University, Aljouf, Saudi Arabia

${ }^{8}$ The University of Manchester, School of Physics and Astronomy, Oxford Road, M13 9PL Manchester, United Kingdom

${ }^{9}$ Department of Nuclear Physics and Biophysics, Comenius University in Bratislava, 84248 Bratislava, Slovakia

${ }^{10}$ Department of Physics, University of Liverpool, Liverpool, L69 7ZE, United Kingdom

${ }^{11}$ GSI Helmholtzzentrum für Schwerionenforschung GmbH, Darmstadt 64291, Germany

${ }^{12}$ Center for Nuclear Study (CNS), Graduate School of Science The University of Tokyo, Japan

${ }^{13}$ CSNSM Orsay, 101 Domaine de l'Universit de Paris S, 91400 Orsay, France

${ }^{14}$ Institut für Physik, Universität Greifswald, 17487 Greifswald, Germany

${ }^{15}$ Institut für Physik, Johannes Gutenberg-Universität Mainz, Mainz, D-55128, Germany

${ }^{16}$ Institut für Kern- und Teilchenphysik, Technische Universität Dresden, Dresden 01069, Germany

${ }^{17}$ Institut für Kernphysik, Technische Universität Darmstadt, Darmstadt 64289, Germany

(Received 6 March 2020; accepted 23 July 2020; published 11 August 2020; corrected 20 October 2021)

\begin{abstract}
A study of the ground states of the laser-ionized and mass-separated odd-odd isotopes ${ }^{180,182}$ Au was performed using the Resonance Ionization Laser Ion Source, Windmill detection setup and ISOLTRAP Multi-Reflection Time-of-Flight Mass Spectrometer at ISOLDE, CERN. A complex fine-structure $\alpha$-decay pattern of ${ }^{180} \mathrm{Au}$ was deduced, providing insight into the low-lying levels in the daughter nucleus ${ }^{176} \mathrm{Ir}$. An $\alpha$-decay branching ratio of $b_{\alpha}\left({ }^{180} \mathrm{Au}\right)=0.58(10) \%$ and a half-life of $T_{1 / 2}=7.2(5) \mathrm{s}$ have also been derived, allowing for the calculation of the reduced $\alpha$-decay widths and determining the degree of hindrance of respective $\alpha$-decay branches. From complementary first in-source laser spectroscopy measurements of the hyperfine structure in atomic transitions of ${ }^{180,182} \mathrm{Au}$, the nuclear magnetic moments of $\mu\left({ }^{180} \mathrm{Au}\right)=-0.83(9) \mu_{N}$ and $\mu\left({ }^{182} \mathrm{Au}\right)=1.66(9) \mu_{N}$ were extracted with an inclusion of a correction for the hyperfine anomaly. Based on the observed hyperfine structure patterns, and on the comparison of the measured and calculated $\mu$ values, a preferred ground-state spin and parity $I^{\pi}\left({ }^{180} \mathrm{Au}^{\mathrm{gs}}\right)=\left(1^{+}\right)$is proposed, and the earlier assignment of $I^{\pi}\left({ }^{182} \mathrm{Au}^{\mathrm{gs}}\right)=\left(2^{+}\right)$is confirmed. For ${ }^{180} \mathrm{Au}$, the
\end{abstract}

\footnotetext{
*andrei.andreyev@york.ac.uk

${ }^{\dagger}$ Present address: CERN, 1211, Geneva 23, Switzerland.

†Present address: TRIUMF, Vancouver BC V6T 2A3, Canada.

§Present address: Centre d'Etudes Nuclaires de Bordeaux-Gradignan, 19 Chemin du Solarium, CS 10120, F- 33175 Gradignan, France.

"Present address: Belgian Nuclear Research Center SCKCEN, Boeretang 200, B-2400 Mol, Belgium.

IPresent address: Universit Paris-Saclay, CNRS/IN2P3, IJCLab, 91405 Orsay, France.

\#Present address: ARC Centre of Excellence for Engineered Quantum Systems, The University of Sydney, NSW 2006, Australia.
}

Published by the American Physical Society under the terms of the Creative Commons Attribution 4.0 International license. Further distribution of this work must maintain attribution to the author(s) and the published article's title, journal citation, and DOI. 
most probable proton-neutron Nilsson configuration of $\pi 3 / 2^{-}[532] \otimes v 5 / 2^{-}[512]$ suggests the same proton state as in the heavier deformed odd-odd nuclei ${ }^{182,184} \mathrm{Au}$.

DOI: 10.1103/PhysRevC.102.024312

\section{INTRODUCTION}

Understanding the structural evolution of the ground and isomeric states in nuclei very far from stability is one of the challenges faced by current experimental and theoretical efforts in nuclear physics research. Historically, the neutrondeficient lead region has been one of the richest playgrounds for a variety of nuclear structure phenomena, with shape coexistence and the presence of deformed intruder states at low excitation energies being just some of the examples [1-5].

Since the 1980s, the neutron-deficient gold isotopes $(Z=$ 79) have played an important role in studies of the shape coexistence in this region. Hyperfine structures (hfs) and isotopic shifts (IS) measurements down to the neutron midshell at $N=104$ revealed a sudden change from nearly spherical to strongly deformed shape for the ground states (g.s.) of ${ }^{183-186} \mathrm{Au}(N=104-107)$, compared to heavier gold isotopes [6-9]. Electric quadrupole moment measurements for the long-lived ground and isomeric states in ${ }^{184} \mathrm{Au}$, being the lightest gold isotope for which the quadrupole moment was measured so far, provided direct evidence of their prolate deformation [10]. This shape change was interpreted as being due to the occupation change from the spherical $\pi 3 s_{1 / 2} / \pi 2 d_{3 / 2}$ orbitals responsible for the g.s. of the heavier isotopes, to prolate-deformed $\pi 3 / 2^{-}$[532] and/or $\pi 1 / 2^{-}$[541] Nilsson states of $h_{9 / 2}$ parentage when approaching the neutron midshell [10-12].

Following recent advances in the decay-tagged in-source laser-spectroscopy technique [13], a dedicated program to study the ground- and isomeric-state properties of the neutrondeficient gold isotopes has been undertaken by our collaboration at the CERN-ISOLDE facility [14]. The first results from this campaign include the determination of spins and magnetic dipole moments of the g.s. for ${ }^{177,179} \mathrm{Au}$ [15], the discussion of the hyperfine anomaly for the $11 / 2^{-}$isomeric states in ${ }^{177,191,193,195} \mathrm{Au}$ [16], and of shape coexistence in ${ }^{187} \mathrm{Au}$ [17]. In the present work we report on a study of odd-odd isotopes ${ }^{180,182} \mathrm{Au}(N=101,103)$, which are located in the expected region of large ground-state deformation in the neutron-deficient gold nuclei [18].

The known $\alpha$-decay spectroscopy data for ${ }^{180}$ Au originate from experiments at the SHIP velocity filter at GSI by Keller et al. [19] and the GSI on-line mass separator, by Wauters et al. [20]. As both studies used complete-fusion reactions to produce ${ }^{180} \mathrm{Au}$ nuclei, several other nuclides originating from different $(x n),(p, x n)$, and $(\alpha, x n)$ evaporation channels were implanted in the detection systems, despite the use of the respective separators. The application of such mixed beams resulted in some limitations in the measurements of decay properties. The SHIP investigation attributed a single $E_{\alpha}=$ $5685(10) \mathrm{keV}$ decay to ${ }^{180} \mathrm{Au}$ and deduced a lower limit on the $\alpha$-decay branching ratio, $b_{\alpha}\left({ }^{180} \mathrm{Au}\right) \geqslant 1.8 \%$. However, this decay was not observed in the work by Wauters $e t$ al.
[20]. Instead, at least seven $\alpha$ decays were seen for ${ }^{180} \mathrm{Au}$, but only three of them [5497(10), 5611(10), and 5648(10) keV] were placed in the tentative decay scheme (see Fig. 10 in Ref. [20]). Four $\gamma$ rays $(36.5,41.5,118$, and $195 \mathrm{keV})$ were seen in coincidence with these $\alpha$ decays. No information on the relative intensities of $\alpha$ decays or $\gamma$-ray multipolarities was provided.

The properties of the g.s. and of low-energy excitations in $\alpha$-decay daughter nuclide ${ }^{176} \mathrm{Ir}$ are not well established. Several $\beta-\gamma$ decay studies of ${ }^{176}$ Ir have been performed [21-23]. In particular, based on the predominant feeding of $I^{\pi}=4^{+}$and $6^{+}$states in the $\beta$-decay daughter ${ }^{176} \mathrm{Os}$, the study [21] proposed a most-likely spin-parity of $I=\left(5^{+}\right)$for the 8(1) s ground state of ${ }^{176} \mathrm{Ir}$, see the summary in NNDC [24]; the latter evaluation, however, refrains from providing the spin value for the g.s. of ${ }^{176} \mathrm{Ir}$. This could be due to the fact that, while the latest $\beta$-decay investigation of ${ }^{176} \operatorname{Ir}$ [23] confirmed the findings of the previous studies, it also proposed the existence of a low-spin longer-lived 17.6(17) s isomeric state in this nucleus, with most probable spin of 2 or 3 . No information on the relative position of the above-mentioned states in ${ }^{176} \mathrm{Ir}$ was provided, thus it is not clear which of them is the ground state. Furthermore, a rich pattern of several floating high-spin bands with band heads of $I^{\pi}=5^{+} / 7^{+} / 8^{-}$ was observed in ${ }^{176}$ Ir by in-beam spectroscopy (see Fig. 1 of Ref. [25]). Importantly, the complex low-lying level structure in ${ }^{176} \mathrm{Ir}$, as suggested by calculations in Ref. [23], might already give a clue to the complex fine-structure (f.s.) $\alpha$-decay pattern of ${ }^{180} \mathrm{Au}$ observed by Wauters et al. [20].

The present study of ${ }^{180} \mathrm{Au}$ at ISOLDE contains $\approx 10$ times more statistics than the work by Wauters et al. [20]. In a combination with the purity of the sample provided by the laser ionization, these statistics have allowed us to derive a more detailed decay scheme for ${ }^{180} \mathrm{Au}$. The paper is organized in the following way. Section II gives a brief overview of the experimental techniques. Section III A 1 describes the $\alpha$ decay of ${ }^{180} \mathrm{Au}$, while discussions of $\beta$ decay and hfs are presented in Secs. III B and III C, respectively; the latter also includes the hfs data for ${ }^{182} \mathrm{Au}$. The extracted magnetic dipole moments for ${ }^{180,182} \mathrm{Au}$ are used in Sec. IV A, together with Nilsson model calculations, to deduce the preferred g.s. spin and configuration for ${ }^{180,182} \mathrm{Au}$. The systematics of the reduced widths and hindrance factors for $\alpha$ decays of ${ }^{180} \mathrm{Au}$ are discussed in Sec. IV B.

\section{EXPERIMENT}

The present data originate from the same experimental campaign as described in Refs. [15-17], whereby ${ }^{180,182} \mathrm{Au}$ nuclei were produced in spallation reactions induced by a 1.4-GeV proton beam from the CERN PS Booster, impinging on a $50 \mathrm{~g} / \mathrm{cm}^{2}$ thick $\mathrm{UC}_{\mathrm{x}}$ target. A proton beam current of up to $2 \mu \mathrm{A}$ was used. The spallation products diffused out of the 
hot target $(T \approx 2500 \mathrm{~K})$ and effused as neutral atoms into the hot ion cavity of the Resonance Ionisation Laser Ion Source (RILIS) [26]. The gold atoms were ionized within this cavity when the laser beams were frequency tuned to the three-step gold ionization scheme, as was described in Ref. [15], see further details in Sec. III C.

After selective ionization, the ions were accelerated by a $30-\mathrm{kV}$ electrostatic potential, and mass separated according to their mass-to-charge ratio using the General Purpose Separator (GPS). The pure ion beam of the isotope of interest was then transported either to the Windmill (WM) decay station [27] or the ISOLTRAP Multi-Reflection Time-of-flight Mass Spectrometer (MR-ToF MS) [17,28]. The WM detection system was utilized for detailed $\alpha$ - and $\beta$-decay studies of ${ }^{180} \mathrm{Au}$, while both setups were used for taking hfs scans for ${ }^{180,182} \mathrm{Au}$, either via $\alpha$-decay tagging in the case of the WM or photo-ion counting using the high-resolution mass-selective MR-ToF MS, see Refs. [13,17,29] for further details on the scanning procedure.

The WM detection system used in this study is shown in Fig. 1 of Ref. [27], here only a short description is given. The ion beam passes through a central hole of an annular surface-barrier silicon detector ( $\mathrm{Si} 1)$ and is implanted into one of ten thin carbon foils of $20 \mu \mathrm{g} / \mathrm{cm}^{2}$ thickness, mounted on a rotatable wheel. A second surface-barrier silicon detector (Si2) is positioned $\approx 5 \mathrm{~mm}$ behind the implantation foil. The total $\mathrm{Si} 1+\mathrm{Si} 2$ detection efficiency for $\alpha$ particles was $28(3) \%$ at this implantation position. A second pair of PIPS silicon detectors ( $\mathrm{Si3}$ and $\mathrm{Si} 4$ ) was located at the decay position, four foil positions away from the implantation point. They measured $\alpha$ decays from the longer-lived daughter products of each gold isotope after periodic movement of the wheel, which brought the remaining activity from the implantation to the decay position. The total $\mathrm{Si} 3+\mathrm{Si} 4$ detection efficiency was $38(2) \%$. The silicon detectors were calibrated individually, see Sec. III A for details. Typical full width half maximum (FWHM) energy resolutions of 25 and $30 \mathrm{keV}$ were observed for $\alpha$ decays in the energy region of 5-6 MeV for Sil and $\mathrm{Si} 2-4$, respectively.

A single-crystal low-energy germanium detector (LEGe) with a thin beryllium window was placed outside the WM chamber behind the Si2 detector, along the axis of the beam. The WM aluminum back wall had a thickness of $1 \mathrm{~mm}$ at this position, allowing $\gamma$ rays with energies down to $\approx 30 \mathrm{keV}$ to be measured. The typical FWHM energy resolution and detection efficiency of the LEGe at $121.8 \mathrm{keV}$ was $1.1 \mathrm{keV}$ and $7.7 \%$, respectively. A high-purity germanium (HPGe) detector with a typical energy resolution of $2.3 \mathrm{keV}$ at $1178 \mathrm{keV}$ was placed outside the WM chamber orthogonally to the implantation point. Both detectors were calibrated energywise and efficiencywise using ${ }^{152} \mathrm{Eu},{ }^{137} \mathrm{Cs},{ }^{133} \mathrm{Ba}$, and ${ }^{60} \mathrm{Co}$ sources. Due to the side placement of the HPGe detector behind the $1-\mathrm{cm}$ thick wall of the WM chamber, its $\gamma$-ray detection efficiency was much lower than that of the LEGe, especially for low energies. Thus, the HPGe detector was mostly used to study the $\gamma-\gamma$ coincidences related to the decay of ${ }^{180} \mathrm{Au}$, discussed in Sec. III B.

\section{RESULTS}

\section{A. $\alpha$ decay of ${ }^{180} \mathrm{Au}$}

A summed singles $\alpha$-decay energy spectrum registered in Si1 and Si2 is shown in Fig. 1(a), with the strongest $\alpha$-decay peaks belonging to ${ }^{180} \mathrm{Au}$ and its $\beta$-decay daughter ${ }^{180} \mathrm{Pt}$. A total number of $\alpha$ decays of ${ }^{180} \mathrm{Au}$ observed in the $\alpha$-energy region of $5300-5700 \mathrm{keV}$ is $6.4 \times 10^{4}$. It is important to note here that the $\alpha$-decay energies quoted for ${ }^{180} \mathrm{Au}$ in Fig. 1(a), and further in the text and in the decay scheme are the values after a small correction $(\approx 1-6 \mathrm{keV})$ for $\alpha+$ conversion electrons summing in the silicon detectors was implemented, based on GEANT4 simulations, as discussed in Sec. III A 4.

Due to the relatively low resolving power of the GPS, a weak $\alpha$-decay peak from the neighboring mass ${ }^{179} \mathrm{Au}$ is also seen in the spectrum, with $\approx 1.7 \times 10^{3}$ times lower intensity. ${ }^{180} \mathrm{Hg}$ and its $\alpha$-decay daughter ${ }^{176} \mathrm{Pt}$ are also weakly present, as products from the decay chain of surface-ionized nuclide ${ }^{180} \mathrm{Tl}$. Their decays aid in the validation of the calibration procedure outlined below.

The energy calibration of the silicon detectors also accounted for possible energy shifts and/or broadening of $\alpha$ decay peaks due to the recoil effects after $\alpha$ decay of the external activity implanted in a thin carbon foil, see, e.g., Ref. [31]. To avoid these issues, in the energy calibration procedure we used only $\alpha$ decays of the parent nuclei and of their $\beta$-decaying daughters, as the latter do not suffer from the recoil effect.

For ${ }^{180} \mathrm{Au}$, the energy calibration was performed using the $\alpha$ decays from ${ }^{178} \mathrm{Pt}$ (5446(3) $\mathrm{keV}$ [32]) and ${ }^{179} \mathrm{Au}$ (5848(5) $\mathrm{keV}$ [33]), taken from short measurements at the respective GPS mass settings before the main run at $A=180$. This procedure was complemented with the use of $\alpha$ decays of ${ }^{180} \mathrm{Pt}\left[5160(5) \mathrm{keV}^{1}\right.$ ] and ${ }^{180} \mathrm{Hg}(6119$ (4) $\mathrm{keV}$ [36]) weakly present in the $A=180 \alpha$-decay spectrum in Fig. 1(a).

Based on this calibration, we deduced a small shift by $\approx 9-12 \mathrm{keV}$ to lower values of the $\alpha$-decay energies of ${ }^{180} \mathrm{Au}$ compared to the previous study by Wauters et al. [20]. For example, in Fig. 1(a) the highest-energy $\alpha$ decay attributed to ${ }^{180} \mathrm{Au}$ is observed at $5639(7) \mathrm{keV}$, compared to $5648(5)$ $\mathrm{keV}$ quoted in Ref. [20]. Most likely, this shift stems from the difference in $\alpha$-decay energies used for calibration. In Ref. [20], the 5458-keV decay of ${ }^{178} \mathrm{Pt}$ was used for calibration, $12 \mathrm{keV}$ higher than the presently accepted literature value of 5446(3) keV [32].

Apart from the main $5160-\mathrm{keV}$ g.s. $\rightarrow$ g.s. decay of ${ }^{180} \mathrm{Pt}$, a weak $\alpha$ decay at 5028(7) $\mathrm{keV}$ is seen in Fig. 1(a), which is attributed to the f.s. decay of ${ }^{180} \mathrm{Pt}$ to the first $2^{+}$state in ${ }^{176}$ Os at $135.1 \mathrm{keV}$. This decay was already shown in Fig. 7(a) of Ref. [20], but to our knowledge its energy and intensity have never been reported in the literature. Based on the data

\footnotetext{
${ }^{1}$ This value was recently deduced in our study at ISOLDE [34], and deviates slightly from the value of $5140(10) \mathrm{keV}$ reported in the 1966 work [35].
} 

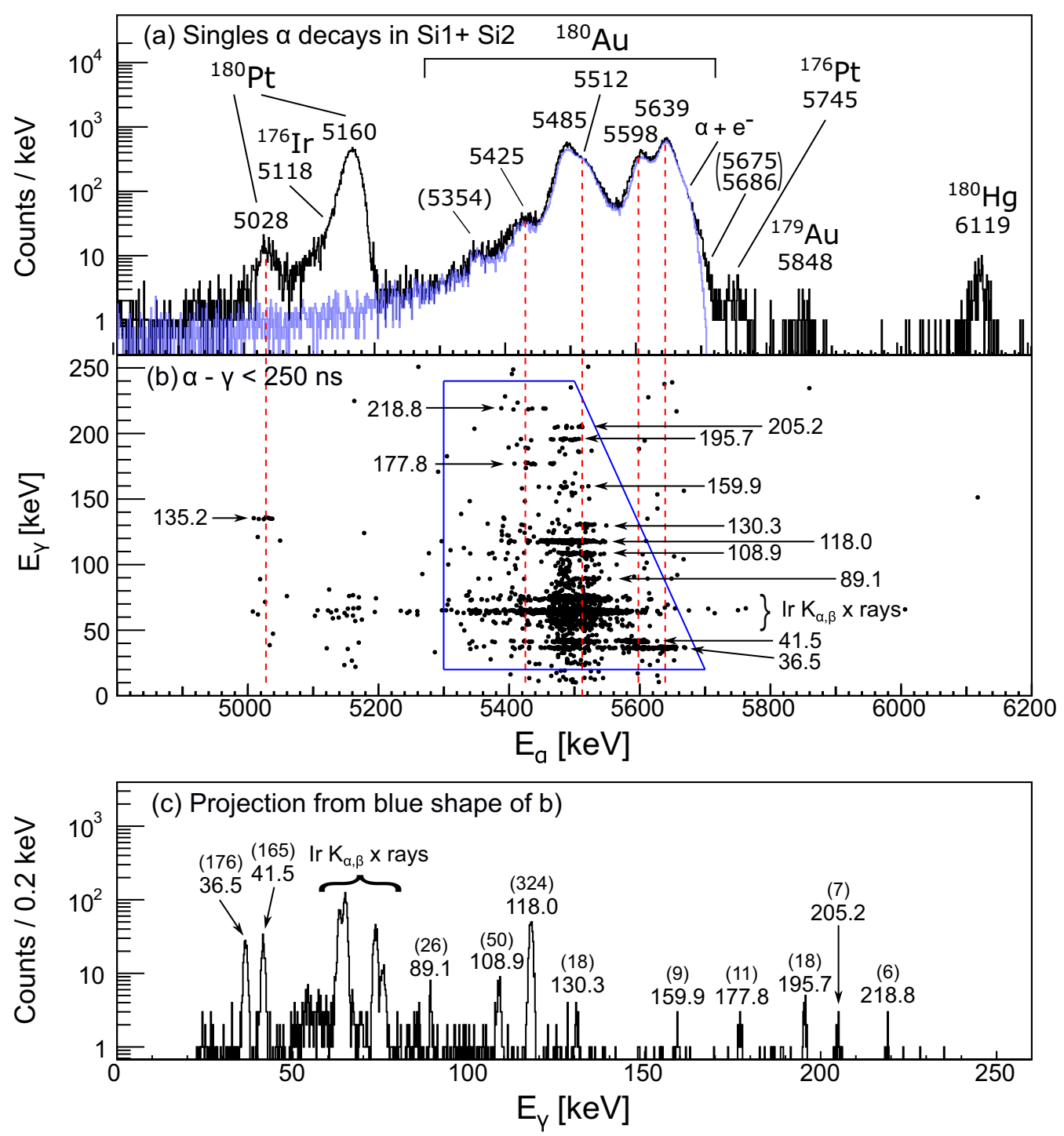

FIG. 1. (a) Singles $\alpha$-decay spectrum registered in Si1 and Si2 at $A=180$; the peaks are marked with their energies in keV. The quoted $\alpha$-decay energies for ${ }^{180} \mathrm{Au}$ include the correction for $\alpha+$ conversion electrons summing in silicon detectors. The overlapped blue histogram is the result of GEANT4 simulations, see Sec. III A 4 for details. (b) $\alpha-\gamma$ coincidences for $\alpha$ decay from (a) for $\gamma$-ray energies up to 250 keV with a prompt coincidence timing gate $\Delta T(\alpha-\gamma)<250 \mathrm{~ns}$. (c) Projection on the $\gamma$-ray axis for events inside the blue region in (b). The $\gamma$-ray peaks in (b) and (c) are marked with their energies in keV. Additionally, the number of counts in each peak is given in brackets in (c).

in Fig. 1(a), the intensity of this f.s. decay was deduced as $2.2(2) \%$.

\section{1. $\alpha-\gamma$ coincidences for ${ }^{180} \mathrm{Au}$}

An $\alpha-\gamma$ coincidence matrix for prompt $\gamma$-ray transitions registered in the LEGe following $\alpha$ decays measured in $\mathrm{Si} 1 / \mathrm{Si} 2$, is shown in Fig. 1(b). A prompt time gate of $\Delta T(\alpha-$ $\gamma)<250 \mathrm{~ns}$ was used. A projection on the $E_{\gamma}$ axis for the events within the blue-shaped region in Fig. 1(b), which is relevant for ${ }^{180} \mathrm{Au}$, is shown in Fig. 1(c). It contains several low-energy transitions with energies up to $218 \mathrm{keV}$, along with a large number of iridium $K_{\alpha, \beta}$ x rays. The $\gamma$ rays at 36.5(3) $\mathrm{keV}, 41.5(3) \mathrm{keV}, 118.0(3) \mathrm{keV}$, and 195.7(5) keV were previously reported by Wauters et al. [20], while the transitions at 89.1(4), 108.9(4), 130.3(4), 159.9(5), 177.8(4), 205.2(5), and 218.8(5) keV are newly observed.

Several f.s. $\alpha$ decays of ${ }^{180} \mathrm{Au}$ were distinguished by gating on coincident $\gamma$ rays from Fig. 1(c) and were placed in the decay scheme shown in Fig. 2, based on the arguments presented further in the text. Figure 3 shows the respective $\alpha$-decay projections for some of these $\gamma$ rays.

The 5639(7)-keV $\alpha$ decay is seen in coincidence with a 36.5-keV $\gamma$ ray, see Fig. 3(a). This establishes an excited state at $36.5(3) \mathrm{keV}$ in ${ }^{176} \mathrm{Ir}$, as depicted in the decay scheme shown in Fig. 2. Based on this $\alpha-\gamma$ coincidence pair, we define the reference full energy $Q_{\alpha, \text { ref }}=Q_{\alpha}(5639)+E_{\gamma}(36.5)=$ $5804(7) \mathrm{keV}$, which will be used throughout the text.

An $\alpha$ decay at 5598(8) $\mathrm{keV}$ is seen in coincidence with both the $41.5-\mathrm{keV}$ and the $36.5-\mathrm{keV} \gamma$ rays, see Figs. 3(a), 3(b). The value $Q_{\alpha \text {,tot }}=Q_{\alpha}(5598)+E_{\gamma}(41.5)$ 


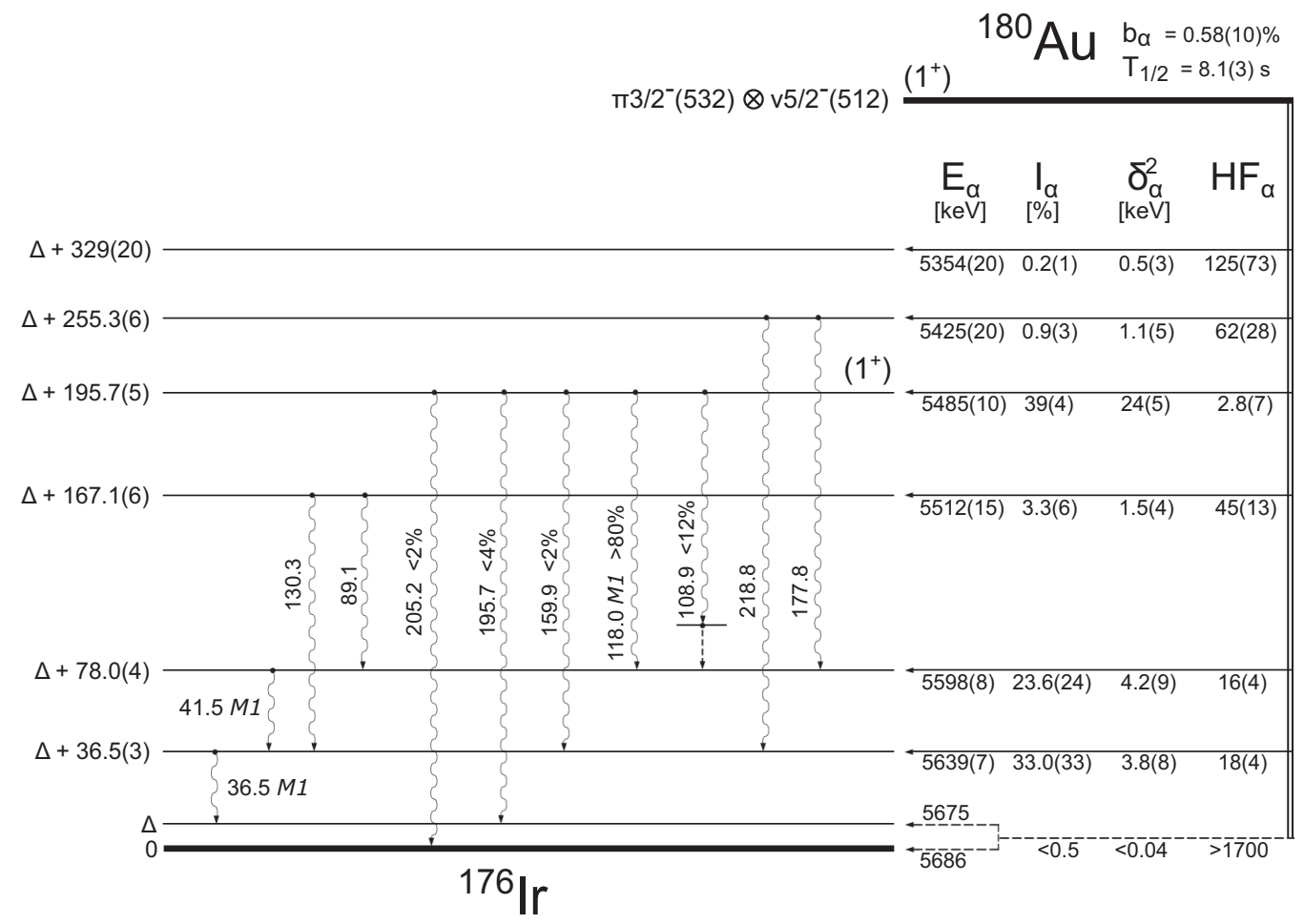

FIG. 2. A decay scheme for ${ }^{180} \mathrm{Au}$ proposed in this study. The $\alpha$-decay energies $E_{\alpha}$, relative intensities $I_{\alpha}$, reduced $\alpha$-decay widths $\delta_{\alpha}^{2}$ and hindrance factor values $\mathrm{HF}_{\alpha}$ for the parent isotope ${ }^{180} \mathrm{Au}$ and levels and transitions in the daughter nucleus ${ }^{176}$ Ir deduced in this work are shown alongside some previously-known data from Ref. [20]. The half-life value of 8.1(3) s is taken from [30]. Hindrance factors were extracted relative to the average value of $\delta_{\alpha}^{2}=66(6) \mathrm{keV}$ for the unhindered $\alpha$ decays from ${ }^{179,181} \mathrm{Au}$, see Sec. IV B for details. A value $\Delta=9.5(7) \mathrm{keV}$ is discussed in Sec. III A 2. The 5675-keV and 5686-keV $\alpha$ decays are shown by a dashed line as tentative.

$+E_{\gamma}(36.5)=5803(8) \mathrm{keV}$ matches well to $Q_{\alpha, \text { ref. }}$ This proves that the $41.5-\mathrm{keV}$ and $36.5-\mathrm{keV} \gamma$ rays form a $\gamma$-ray cascade following the 5598-keV $\alpha$ decay, establishing a level at $78.0(4) \mathrm{keV}$ as already proposed in the study by Wauters et al. [20].

An $\alpha$ decay at $5485(10) \mathrm{keV}$ is observed in coincidence with 36.5-, 41.5-, and 118-keV $\gamma$ rays, see projections in Figs. 3(a), 3(b), and 3(e). Due to the matching of $Q_{\alpha, \text { tot }}=Q_{\alpha}(5485)+E_{\gamma}(118)+E_{\gamma}(41.5)+E_{\gamma}(36.5)=$ $5806(10) \mathrm{keV}$ to the $Q_{\alpha, \text { ref }}$, the $118-\mathrm{keV}$ transition is assigned in a cascade with the $41.5-\mathrm{keV}$ and $36.5-\mathrm{keV} \gamma$ rays. The $195.7-\mathrm{keV} \gamma$ ray, which is also seen in coincidence with the 5485-keV decay [see Fig. 3(g)] is placed as a crossover transition to the same state in ${ }^{176} \mathrm{Ir}$ as fed by the $36.5-\mathrm{keV}$ decay. This confirms a level at $195.7(5) \mathrm{keV}$ in ${ }^{176} \mathrm{Ir}$, which has been proposed in Ref. [20]. Within the experimental energy uncertainties, the weak $159.9-\mathrm{keV}$ transition seen in Fig. 1(c) matches well to the decay between the 195.7- and 36.5-keV levels, thus it was also placed in the decay scheme in Fig. 2.

The $5485-\mathrm{keV}$ decay is also seen in coincidence with the 108.9-keV $\gamma$ ray, see Fig. 3(d). This observation might suggest that an $\approx 9 \mathrm{keV}$ transition should exist in a cascade with the $108.9 \mathrm{keV}$ decay, but it would be unobserved in our experiment. The 5485-keV peak in Fig. 3(d) has a higherenergy tail, which tentatively might be attributed as being due to the summing of the $5485-\mathrm{keV}$ transition with the conversion electrons originating from this 9-keV decay, also partially from summing with electrons from 36.5- and 41.5$\mathrm{keV}$ decays, see Sec. III A 4. We tentatively placed the 108.9keV decay as shown in Fig. 2, followed by the yet unobserved $\approx 9 \mathrm{keV}$ decay, which is shown by a dashed arrow as tentative.

An $\alpha$ decay of 5512(15) $\mathrm{keV}$ is in coincidence with the 89.1- and 130.3-keV $\gamma$ rays, see Figs. 3(c) and 3(f), respectively. The $Q_{\alpha \text {,tot }}=Q_{\alpha}(5512)+E_{\gamma}(89.1)+E_{\gamma}(41.5)+$ $E_{\gamma}(36.5)=5804(15) \mathrm{keV}$ is in good agreement with $Q_{\alpha, \text { ref }}$. Thus, the $89.1-\mathrm{keV} \gamma$ ray is placed in cascade with the 41.5and $36.5-\mathrm{keV} \gamma$ rays, establishing a level at 167.1(6) $\mathrm{keV}$. The $130.3-\mathrm{keV} \gamma$ ray is assigned as a crossover transition feeding the $36.5-\mathrm{keV}$ level.

A weak $\alpha$ decay at $5425(20) \mathrm{keV}$ is seen in singles $\alpha$-decay spectrum in Fig. 1(a). It is also seen in coincidence with 218.8-keV and the $177.8-\mathrm{keV} \gamma$ rays, see Fig. 3(i). Since the value of $Q_{\alpha \text {,tot }}=Q_{\alpha}(5425)+E_{\gamma}(218.8)+E_{\gamma}(36.5)=$ $5803(20) \mathrm{keV}$ is in agreement with $Q_{\alpha, \text { ref }}$, the $218.8-\mathrm{keV} \gamma$ ray is placed in cascade with the $36.5-\mathrm{keV}$ transition, establishing a level at 255.3(6) $\mathrm{keV}$. The $177.8-\mathrm{keV} \gamma$ ray is proposed as a transition to the $78-\mathrm{keV}$ level, as the $\gamma$-ray energy difference $218.8(5)-177.8(5)=41.0(7) \mathrm{keV}$ fits well to the energy of 41.5(3)-keV decay from this level.

In Fig. 1(a), a weak $\alpha$ decay at $5354(20) \mathrm{keV}$ is seen in coincidence with the $\operatorname{Ir} K_{\alpha, \beta}$ x rays, see Fig. 1(b). The latter confirms that the 5354-keV decay originates from ${ }^{180} \mathrm{Au}$. The $Q_{\alpha}$ analysis requires the presence of an excited state at 


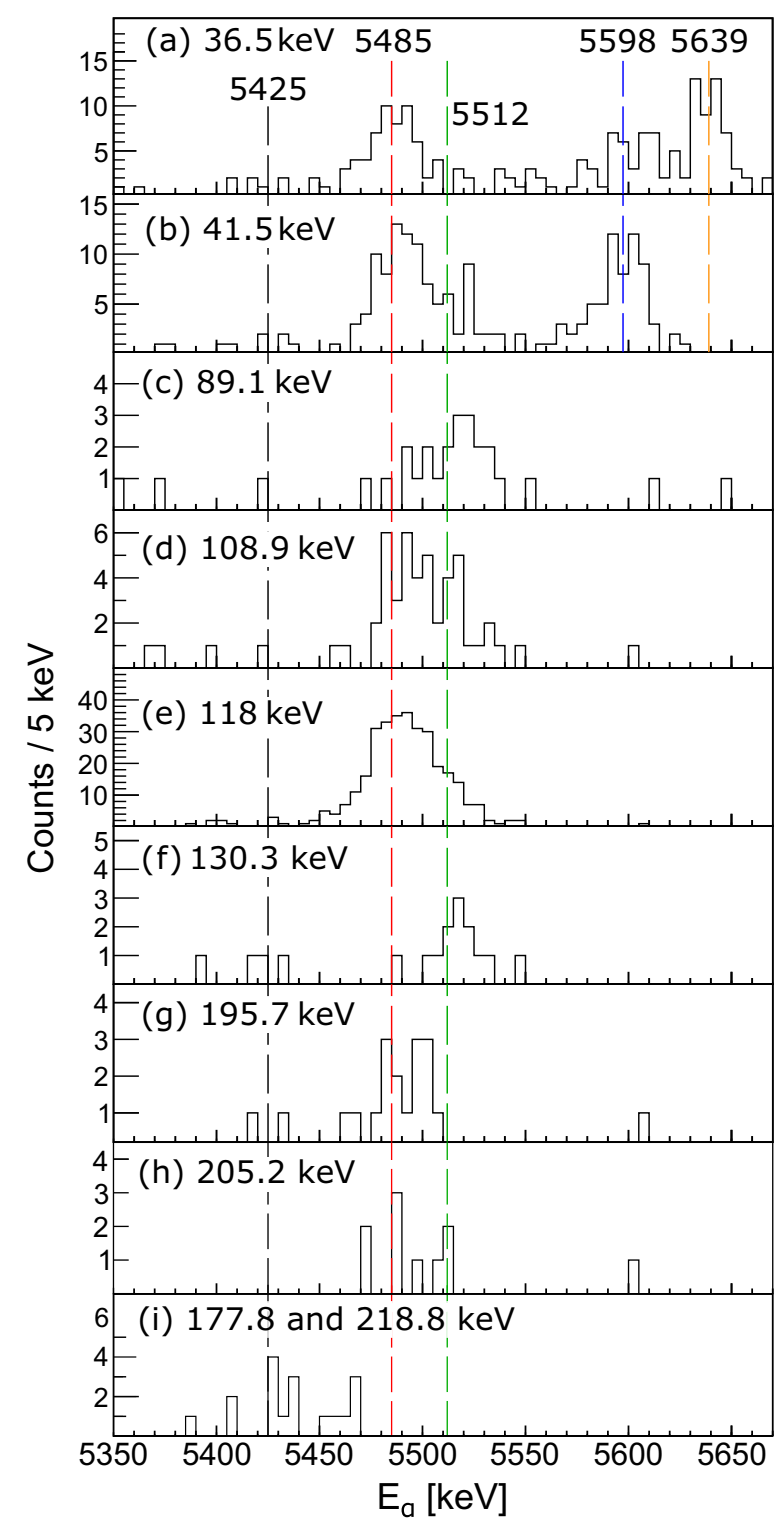

FIG. 3. Projections on the $\alpha$-energy axis from $\alpha-\gamma$ coincident plot in Fig. 1(b), using a $\pm 1.5 \mathrm{keV}$ gate encompassing the $\gamma$ ray indicated in the top left of each projection.

$E^{*}=329(20) \mathrm{keV}$ in ${ }^{176} \mathrm{Ir}$, but no coincident $\gamma$-ray transition or a cascade could be identified following this weak $\alpha$ decay. The number of $\mathrm{Ir} K \mathrm{x}$ rays in coincidence with this decay suggests that such a transition (or a cascade of them) should be strongly converted, possibly implying its (their) $M 1$ and/or $E 0$ component, and also possibly proceeding via some known lower-lying states, which deexcite via partially converted transitions. This $\alpha$ decay and respective excited state were added to the decay scheme in Fig. 2.

\section{Determination of the $\Delta$ value in the decay scheme of ${ }^{180} \mathrm{Au}$}

Finally, as shown in Figs. 1(b) and 3(h), the 5485-keV decays are also seen in coincidence with the 205.2-keV $\gamma$ ray. In this case, the $Q_{\alpha \text {,tot }}=Q_{\alpha}(5485)+E_{\gamma}(205.2)=$ $5815(10) \mathrm{keV}$, thus it is $\approx 11 \mathrm{keV}$ higher than the $Q_{\alpha \text {,ref }}$ value, albeit consistent within a large uncertainty. Furthermore, the energy difference for the 205.2(5)- and 195.7(5)-keV $\gamma$ rays, which follow the $5485-\mathrm{keV}$ decay, determines a more precise value of $\Delta=9.5(7) \mathrm{keV}$, shown in the decay scheme in Fig. 2. Such a scenario supports the decay scheme proposed by Wauters et al. in Ref. [20], where $\Delta$ was introduced (see Fig. 10 in Ref. [20]), albeit with no prescribed value or any explanation of why it was introduced.

An attempt was also made to derive an estimate of $\Delta$ based on a comparison of directly measured masses of ${ }^{180} \mathrm{Au}$ and ${ }^{176} \mathrm{Ir}$. To date, the most precise mass excess of $m\left({ }^{180} \mathrm{Au}\right)=$ $-25627.25(4.95) \mathrm{keV} / \mathrm{c}^{2}$ was reported by the ISOLTRAP Penning-trap study [37]. This value agrees with, but is much more precise, than a mass excess of $-25612(28) \mathrm{keV} / \mathrm{c}^{2}$ from direct measurements at the Experimental Storage Ring (ESR) at GSI [38]. The latter work also published a mass excess $m\left({ }^{176} \mathrm{Ir}\right)=-33839(28) \mathrm{keV} / c^{2}$. Based on a combination of ISOLTRAP-ESR measurements for a pair of ${ }^{180} \mathrm{Au}-{ }^{176} \mathrm{Ir}$, a value of $Q_{\alpha}\left({ }^{180} \mathrm{Au}\right)=5787(29) \mathrm{keV}$ can be derived. Within a rather large experimental uncertainty, dominated by the uncertainty of the ESR measurements, this value fits well to $Q_{\alpha}\left({ }^{180} \mathrm{Au}\right)=5802(40) \mathrm{keV}$, if both parent and daughter masses are taken from the ESR data.

Within their large uncertainties, both above-mentioned $Q_{\alpha}\left({ }^{180} \mathrm{Au}\right)$ values match well to the $Q_{\alpha, \text { ref }}=5804(5) \mathrm{keV}$. This analysis also establishes that the $\Delta$ value should not exceed $\approx 20 \mathrm{keV}$, therefore our deduced value of $\Delta=$ 9.5(7) $\mathrm{keV}$ fits to this estimate.

The derivation of $\Delta$ value leads to an important conjecture that, based on the proposed decay scheme and $Q_{\alpha \text {,tot }}$ analysis, two $\alpha$ decays of ${ }^{180} \mathrm{Au}$ with the energies of $\approx 5675 \mathrm{keV}$ and $\approx 5686 \mathrm{keV}$ could be expected, feeding, respectively, to the $\Delta=9.5(7)-\mathrm{keV}$ state and the lowest state in ${ }^{176} \mathrm{Ir}$. Indeed, a small higher-energy tail of the 5639-keV peak, extending up to $\approx 5690 \mathrm{keV}$ is observed in Fig. 1(a), which might include these two $\alpha$ decays. This possibility will be further discussed in Sec. III A 4.

\section{Multipolarities of $\gamma$ rays in ${ }^{176} \mathrm{Ir}$}

36.5- and 41.5-keV $\gamma$ rays. All $\gamma$-ray transitions following the $\alpha$ decay of ${ }^{180} \mathrm{Au}$ are prompt, which limits their multipolarities to $E 1, M 1$, or $E 2$. In our study, a more precise determination of multipolarities could be performed for some of the transitions based on deduced total internal conversion coefficients $\left(\alpha_{\text {tot }}\right)$. As an example, $\alpha_{\text {tot,exp }}(36.5 \mathrm{keV})$ can be deduced from the expression:

$$
\alpha_{\text {tot,exp }}(36.5)=\frac{N_{\alpha} \epsilon_{\gamma}}{N_{\alpha \gamma}}-1,
$$

where $N_{\alpha}$ is the number of singles $5639-\mathrm{keV} \alpha$ decays from Fig. 1(a), $N_{\alpha \gamma}$ is the number of $\alpha(5639)-\gamma(36.5)$ coincidence events from Fig. $3(\mathrm{a})$, and $\epsilon_{\gamma}(36.5)=7.2 \%$ is the $\gamma$-ray detection efficiency at this energy. By using Eq. (1), an $\alpha_{\text {tot,exp }}(36.5)=22(4)$ is derived, which agrees, within the uncertainty, with the theoretical value of $\alpha_{\text {tot,th }}(M 1)=20.6$ calculated using BRICC [39], see Table I. Thus, the $36.5-\mathrm{keV}$ $\gamma$ ray is assigned with a pure $M 1$ multipolarity. 
TABLE I. A comparison of total experimental $\left(\alpha_{\text {tot,exp }}\right)$ and theoretical $\left(\alpha_{\text {tot,th }}\right)$ internal conversion coefficients for listed $\gamma$ decays. The theoretical values were calculated with BRICC [39].

\begin{tabular}{lcccc}
\hline \hline$E_{\gamma}(\mathrm{keV})$ & $\alpha_{\text {tot, exp }}$ & $\alpha_{\text {tot,th }}(E 1)$ & $\alpha_{\text {tot,th }}(M 1)$ & $\alpha_{\text {tot,th }}(E 2)$ \\
\hline $36.5(3)$ & $22(4)$ & 1.21 & 20.6 & 49.9 \\
$41.5(3)$ & $17(4)$ & 0.85 & 14.1 & 265.5 \\
$118.0(3)$ & $\geqslant 3.4(6)$ & 0.26 & 3.8 & 2.38 \\
\hline \hline
\end{tabular}

A value of $\alpha_{\text {tot,exp }}(41.5 \mathrm{keV})$ was further determined by comparing the intensities of the 41.5- and 36.5-keV $\gamma$ rays in coincidence with the 5598-keV $\alpha$ decay, taken from Figs. 3(b) and 3(a), respectively. Due to 41.5 - and $36.5-\mathrm{keV} \gamma$ rays being in a cascade, both groups should have the same intensity, after correction for conversion and $\gamma$-ray detection efficiency.

The deduced value of $\alpha_{\text {tot,exp }}(41.5)=17(4)$ agrees with the theoretical value of $\alpha_{\text {tot,th }}(M 1)=14.1$ within uncertainty (see Table I). Therefore, the $41.5-\mathrm{keV} \gamma$ ray is also assigned with a pure $M 1$ multipolarity.

The energy of a possible direct crossover $78-\mathrm{keV} M 1$ or $E 2$ transition in coincidence with the $5598-\mathrm{keV} \alpha$ decay is about $2 \mathrm{keV}$ higher than the energy of $\operatorname{Ir} K_{\beta 2}$ x rays, thus it should have been resolved in Figs. 1(b) and 1(c), if it existed. However, no evidence for this transition was found, despite its theoretical total conversion coefficients for either $M 1$ (12.3) or $E 2$ (13.2) multipolarities being lower than for the $36.5-\mathrm{keV}$ and $41.5-\mathrm{keV}$ transitions of $M 1$ multipolarity, while the $\gamma$ ray efficiency at $78 \mathrm{keV}$ is slightly higher than for these two lower-energy decays.

118-keV $\gamma$ ray. As shown in the decay scheme in Fig. 2, several f.s. $\alpha$ decays have been identified between 5450 and $5550 \mathrm{keV}$. Of the $\gamma$ rays seen in coincidence with these decays, the 118-keV $\gamma$-ray transition has the largest intensity, see Fig. 1(c). A lower limit on $\alpha_{\text {exp,tot }}(118 \mathrm{keV})$ can be calculated by assuming an $M 1$ multipolarity (thus the largest total internal conversion for $\gamma$ rays at these energies) for the remaining $\gamma$-ray transitions $(130.3,89.1,108.9,159.9$, and $195.7 \mathrm{keV}$ ) in coincidence with the $\alpha$ decays in the 5450-5550 $\mathrm{keV}$ region. By applying the Eq. (1) to this case, the number of $\alpha$ decays preceding each of the $\gamma$ rays listed above was deduced, and subtracted from the total number of $\alpha$ decays in the $5450-5550 \mathrm{keV}$ region. The remaining number gives a lower limit for the number of $\alpha$ decays, $N_{\alpha}(5450-5550$ $\mathrm{keV}$ ), which are followed by a $118-\mathrm{keV} \gamma$ ray or associated conversion electrons.

Using this $N_{\alpha}(5450-5550 \mathrm{keV})$ number in Eq. (1), an $\alpha_{\text {tot,exp }}(118 \mathrm{keV}) \geqslant 3.4(6)$ was deduced, being between the theoretical values of $\alpha_{\text {tot,th }}(M 1)=3.8$ and $\alpha_{\text {tot,th }}(E 2)=2.38$. Due to the prompt character of the $118-\mathrm{keV}$ decay, an $M 2$ or higher multipolarity is excluded. Being a lower limit on $\alpha_{\text {tot }}(118 \mathrm{keV})$, this implies that the $118-\mathrm{keV} \gamma$ ray is predominantly $M 1$ with a possibility for a small $E 2$ admixture. This assignment means that the parities of the states from which the $118-\mathrm{keV}$ and $41.5-\mathrm{keV} \gamma$ rays originate are the same. It also restricts the $159.9-\mathrm{keV}$ and $195.7-\mathrm{keV} \gamma$ rays to either an $M 1$ or $E 2$ character, based on the parities of the states, connected by these decays, as well as accounting for the prompt nature of these transitions.

\section{4. $\alpha-e^{-}$summing in the $\alpha$ decay of ${ }^{180} \mathrm{Au}$}

The presence of strongly converted 36.5-, 41.5-, and 118$\mathrm{keV}$ transitions, which follow most of the observed $\alpha$ decays of ${ }^{180} \mathrm{Au}$, leads to $\alpha+$ conversion electron (CE) summing in the $\mathrm{Si} 1 / \mathrm{Si} 2$ detectors in the WM experiments. Namely, if both the $\alpha$ particle and the prompt CE are registered simultaneously in the same silicon detector, both the original energy and the shape of the $\alpha$-decay peak will be distorted. Depending on the intensity, multiplicity, and energy of the CEs, an energy shift to higher energies, an appearance of a high-energy tail and/or appearance of an artificial peak of full $\alpha+\mathrm{CE}$ energy summing might occur.

To understand these effects, the extensive GEANT4 $[40,41]$ simulations were performed for the WM geometry and including all $\alpha$ decays of ${ }^{180} \mathrm{Au}$. The simulations incorporated all relevant CEs and $x$ rays for the 36.5-, 41.5-, and 118.0$\mathrm{keV} M 1 \gamma$-ray transitions, with the conversion coefficients deduced in the present work (see Table I) and relative intensities of different CE's taken from Ref. [39]. Based on these simulations, an energy shift of $1 \mathrm{keV}$ was deduced for the singlefold $\alpha+\mathrm{CE}(36.5)$ summing, $2 \mathrm{keV}$ for a doublefold $\alpha+\mathrm{CE}(36.5)+\mathrm{CE}(41.5)$ summing, and $6 \mathrm{keV}$ for a triplefold $\alpha+\mathrm{CE}(36.5)+\mathrm{CE}(41.5)+\mathrm{CE}(118)$ summing. Following this procedure, the apparent/uncorrected measured energies of 5640-, 5600-, and 5491-keV of the main peaks of ${ }^{180} \mathrm{Au}$ in Fig. 1(a) were corrected, resulting in the true values of 5639-, 5598-, and 5485-keV, used in the text and in the decay scheme.

A good agreement of the simulated (blue histogram) and measured energy spectra for ${ }^{180} \mathrm{Au}$, by starting from the corrected $\alpha$-decay energies, is shown Fig. 1(a), which confirms the correctness of the applied procedure. The intensities of $\alpha$ decays of ${ }^{180} \mathrm{Au}$ shown in the decay scheme were taken from the GEANT simulations.

A very important fact, relevant to the discussion of $\Delta$ value and hindrance factor values in Sec. IV B, is an experimental observation of a small high-energy tail of the 5639-keV $\alpha$ peak in Fig. 1(a), extending up to $\approx 5690 \mathrm{keV}$. Based on the GEANT simulations, most of this tail can be understood as being due to the full $\alpha+$ CE summing energy of the dominant $L$-shell conversion electron from the $36.5-\mathrm{keV} \gamma$ ray with the 5639-keV $\alpha$ decay, which gives an energy of $5662 \mathrm{keV}$, while the $\approx 4$ times lower $M$-shell conversion summing peak has an energy of $5672 \mathrm{keV}$. Therefore, if real, a small excess of events in the region of 5680-5690 keV could indicate the presence of 5675- and/or 5686-keV $\alpha$ decays of ${ }^{180} \mathrm{Au}$, mentioned in Sec. III A 2. By subtracting the measured and simulated spectra, an upper limit of $I_{\alpha} \leqslant 0.5 \%$ could be established for the combined intensity of these decays, which are schematically shown as a tentative single $\alpha$ decay in Fig. 2; they will be further discussed in Sec. IV B.

To conclude the discussion on the $\alpha+$ CE summing, we also note that the $5685(10)-\mathrm{keV} \alpha$ decay, proposed for ${ }^{180} \mathrm{Au}$ by Keller et al. [19], can now be fully understood as being due to this summing effect. In the SHIP experiment, the ${ }^{180} \mathrm{Au}$ nuclei were implanted to a depth of a few $\mu \mathrm{m}$ into the silicon 


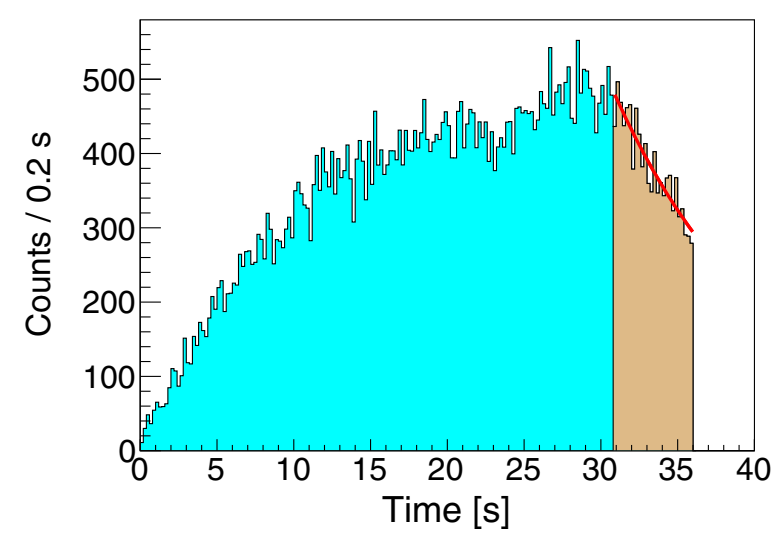

FIG. 4. The time distribution for $\alpha$ decays of ${ }^{180} \mathrm{Au}$ as seen in Si1 and $\mathrm{Si} 2$ at the implantation position. A sequence of implantationdecay cycles was implemented, in which the beam was implanted for $30.9 \mathrm{~s}$ (shaded in blue), followed by the closing of the ISOLDE beam gate for $5.1 \mathrm{~s}$ (shaded in brown). At the end of each $36 \mathrm{~s}$ cycle, the wheel of the Windmill was rotated to place a fresh foil into the implantation position. The decay part of the distribution was fitted with an exponential function, from which a value of $T_{1 / 2}\left({ }^{180} \mathrm{Au}\right)=$ 7.2(5) s was deduced. By extrapolating the decay curve beyond $36 \mathrm{~s}$, the amount of ${ }^{180} \mathrm{Au}$ activity, removed by the wheel movement, can be estimated. The latter is needed for the $b_{\alpha}\left({ }^{180} \mathrm{Au}\right)$ determination.

detector, therefore the degree of $\alpha+\mathrm{CE}$ summing was much higher than in the present study. A dedicated SHIP study by Heßberger et al. [42] provides further examples of importance of $\alpha+\mathrm{CE}$ summing in experiments at recoil separators, employing implantation technique. We performed GEANT simulations for the SHIP-like conditions, which confirmed that the dominant full-energy $\alpha(5598 / 5639 \mathrm{keV})+\mathrm{CE}(36.5,41.5$ $\mathrm{keV}$ ) summing leads to a well-pronounced peak at $\approx 5685$ $\mathrm{keV}$, in agreement with the SHIP data.

\section{Half-life and $\alpha$-decay branching ratio for ${ }^{180} \mathrm{Au}$}

To determine the half-life of ${ }^{180} \mathrm{Au}$, the grow-in-decay-out method, as described in detail in Ref. [43], was applied. The time distribution of the $\alpha$ decays in the energy region 5300 $5700 \mathrm{keV}$ from Fig. 1(a) is presented in Fig. 4. An exponential decay curve is fitted in the $5.1 \mathrm{~s}$ decay time interval between 30.9-36.0 s, from which a half-life of 7.2(5) $\mathrm{s}$ is derived. Within 2-sigma uncertainty, our result is consistent with the previously reported value of 8.1(3) s from Ref. [30]. The precision of the half-life determination in our study is limited by the short time interval $(5.1 \mathrm{~s})$ used in this analysis.

The $\alpha$-decay branching ratio can be derived by comparing the number of $\alpha$ decays from ${ }^{180} \mathrm{Au}$ and its $\beta$-decay daughter ${ }^{180} \mathrm{Pt}$ in Fig. 1(a), as shown in Eq. (2).

$$
\begin{aligned}
b_{\alpha}\left({ }^{180} \mathrm{Au}\right) & =\frac{N_{\alpha}\left({ }^{180} \mathrm{Au}\right)}{N_{\text {tot }}\left({ }^{180} \mathrm{Au}\right)}=\frac{N_{\alpha}\left({ }^{180} \mathrm{Au}\right)}{N_{\alpha}\left({ }^{180} \mathrm{Au}\right)+N_{\mathrm{tot}}\left({ }^{180} \mathrm{Pt}\right)} \\
& =\frac{N_{\alpha}\left({ }^{180} \mathrm{Au}\right)}{N_{\alpha}\left({ }^{180} \mathrm{Au}\right)+\frac{N_{\alpha}\left({ }^{180} \mathrm{Pt}\right)}{b_{\alpha}\left({ }^{180} \mathrm{Pt}\right)}},
\end{aligned}
$$

where $b_{\alpha}\left({ }^{180} \mathrm{Pt}\right)=0.52(5) \%$ [34] and $N_{\text {tot }}$ is the total number of a given isotope produced during the experiment. It is important to note that the direct production of ${ }^{180} \mathrm{Pt}$ is not possible in the experiment. This is due to quasirefractory nature of platinum, resulting in its very long release from the target matrix. Furthermore, platinum surface ionization in the ion source is negligible and it is not ionized with the lasers tuned to the gold atomic transitions. The absence of direct platinum production was demonstrated during the studies of neighboring masses: when the lasers were tuned off the gold ionization scheme, no platinum $\alpha$ decays were observed. Thus, all the ${ }^{180} \mathrm{Pt}$ present in the spectrum can only originate from the $\beta$ decay of implanted ${ }^{180} \mathrm{Au}$.

By direct application of Eq. (2) we determined a branching ratio of $b_{\alpha}\left({ }^{180} \mathrm{Au}\right)=2.2 \%$. However, this value has to be corrected for the periodic movement of the rotatable wheel of the Windmill at the end of each implantation-decay cycle (see also Fig. 4). This movement places the carbon foil with the sample implanted during the current cycle in between the silicon detectors $\mathrm{Si} 3$ and $\mathrm{Si} 4$, where the remaining activity continued to be measured. A full description of respective corrections is provided in Ref. [44]. A $b_{\alpha}=0.58(10) \%$ was deduced for ${ }^{180} \mathrm{Au}$ using Eq. (2), with the intensities of the parent ${ }^{180} \mathrm{Au}$ and daughter ${ }^{180} \mathrm{Pt} \alpha$ decays, corrected for the Windmill movement as mentioned above. This value is about three times smaller than the lower limit of $\geqslant 1.8 \%$ deduced at SHIP [19], however the SHIP value was calculated using a $b_{\alpha}\left({ }^{180} \mathrm{Pt}\right)=0.3 \%$ with a 3-5 factor of uncertainty [45]. In addition, the expression used to calculate $b_{\alpha}\left({ }^{180} \mathrm{Au}\right)$ was incorrect (see Table 2 in Ref. [19]), as confirmed by a private communication with the authors of the experiment.

${ }^{176} \mathrm{Ir}$, being the $\alpha$-decay daughter of ${ }^{180} \mathrm{Au}$, has an $\alpha$ decay with $E_{\alpha}=5118(8) \mathrm{keV}$ [45]. This $\alpha$ decay is masked by the more abundantly produced $5160-\mathrm{keV} \alpha$ decay of ${ }^{180} \mathrm{Pt}$ [see Fig. 1(a)]. However, as this decay branch is small $\left[b_{\alpha}\left({ }^{176} \operatorname{Ir}\right)=\right.$ $3.1(6) \%$ ] [21], its contribution is regarded as negligible in the branching ratio calculation for ${ }^{180} \mathrm{Au}$.

\section{B. ${ }^{180} \mathrm{Au} \beta$ decay}

Figure 5 shows the background-subtracted singles $\gamma$-ray spectrum recorded by the LEGe detector. As no dedicated online measurement of the background was performed during or after the run at $A=180$, we were only able to use a background measurement taken beforehand. This explains the large background still present in Fig. 5, which results from $\gamma$ rays produced in the actual online measurement at $A=$ 180. In this spectrum, the $\gamma$ rays originating from excited states in ${ }^{180} \mathrm{Pt}$ populated by $\beta$ decay of ${ }^{180} \mathrm{Au}$ are labeled, as identified in the previous dedicated $\beta$-decay study of ${ }^{180} \mathrm{Au}$ by Davidson et al. [46]. In particular, the 153.2(1)-keV, $2_{1}^{+} \rightarrow$ $0^{+}$and the 257.5(1)-keV, $4_{1}^{+} \rightarrow 2_{1}^{+} \gamma$ rays from ${ }^{180} \mathrm{Pt}$ are clearly seen. The intensity ratio of $I\left(4_{1}^{+}\right) / I\left(2_{1}^{+}\right) \approx 0.23(5)$ was deduced after the $\gamma$-ray detection efficiency correction. Due to the low statistics, the $346.3-\mathrm{keV}, 6_{1}^{+} \rightarrow 4_{1}^{+}$transition was not observed in our data (see Fig. 5). The decay pattern of feeding the $2_{1}^{+}, 4_{1}^{+}$, and $6_{1}^{+}$states in ${ }^{180} \mathrm{Pt}$ in our work is in agreement with Ref. [46], where the efficiency-corrected ratio of intensities is $I(153.2 \mathrm{keV}) / I(257.5 \mathrm{keV}) / I(346.3$ $\mathrm{keV}) \approx 1000(31): 233(10): 6(2)$, see Table 4 of Ref. [46], thus $I\left(4_{1}^{+}\right) / I\left(2_{1}^{+}\right) \approx 0.23(1)$. 


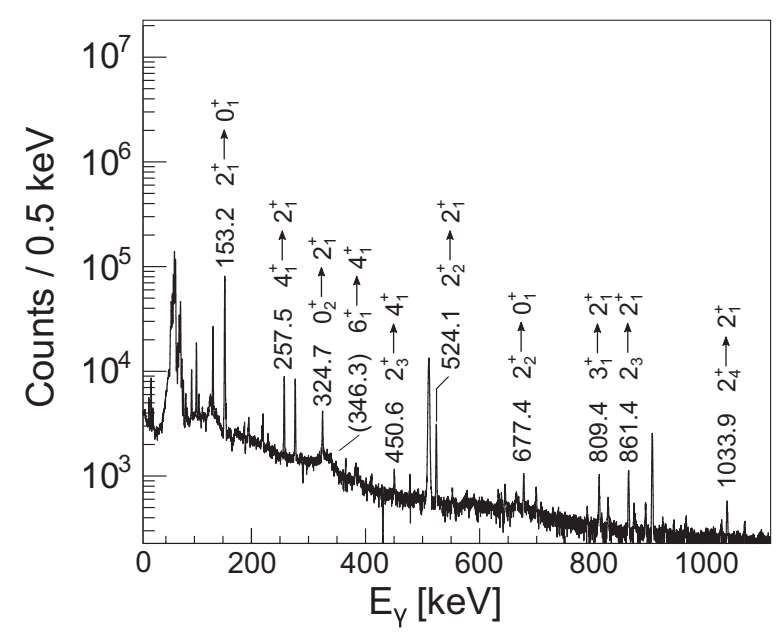

FIG. 5. A background-subtracted $\gamma$-ray energy spectrum detected by the LEGe detector at $A=180$. Transitions associated with the decay of excited levels in ${ }^{180} \mathrm{Pt}$ are indicated (energies in $\mathrm{keV}$ ). The expected position of the unobserved $346.3 \mathrm{keV} 6_{1}^{+} \rightarrow 4_{1}^{+}$decay is indicated in brackets.

As much higher statistics were collected for ${ }^{180} \mathrm{Pt}$ decays in Ref. [46] in comparison to our experiment, in Sec. IV of the present study we will rely on the spin and parity assignments from that work. An apparently weaker intensity for the deexcitation from the $4_{1}^{+}$and, especially, $6_{1}^{+}$states (with respect to $2_{1}^{+}$) is most probably due to the weak feeding from higher-lying states, rather than directly from $\beta$ decay. Indeed, because of the high value of $Q_{\beta}\left({ }^{180} \mathrm{Au}\right)=8.810(12)$ $\mathrm{MeV}$ [47], one can expect some $\beta$-decay feeding to the higherlying states with the observation of subsequent decays usually hampered by the Pandemonium effect. The observed decay pattern suggests an upper limit on the nuclear spin of $I \leqslant 3$ for ${ }^{180} \mathrm{Au}$.

For the followup discussion of hfs measurement in Sec. III C with respect to the spin and configuration of ${ }^{180} \mathrm{Au}$, it is instructive to compare the $\beta$-decay patterns of ${ }^{180,182} \mathrm{Au} \rightarrow$ ${ }^{180,182} \mathrm{Pt}$, presented by Davidson et al. [46]. Similar to ${ }^{180} \mathrm{Pt}$, a predominant feeding of the $2_{1}^{+}$state in ${ }^{182} \mathrm{Pt}$ was also observed in the $\beta$ decay of the known $I=\left(2^{+}\right)$ground state of ${ }^{182} \mathrm{Au}$, see Table 2 in Ref. [46], with the intensity ratios $I(154.9$, $\left.2_{1}^{+}\right) / I\left(264.7,4_{1}^{+}\right) / I\left(355.6,6_{1}^{+}\right) \approx 1000(10): 443(10): 19(5)$, thus $I\left(4_{1}^{+}\right) / I\left(2_{1}^{+}\right) \approx 0.44(1)$. One notices a reduction, of a factor of $\approx 2$, between ${ }^{180,182} \mathrm{Au}$ in the feeding of the $4_{1}^{+}$and $6_{1}^{+}$states compared with the $2_{1}^{+}$state, which may indicate that the ground-state spin of ${ }^{180} \mathrm{Au}$ is lower than that of ${ }^{182} \mathrm{Au}$. The latter inference is based on the fact the structure and positions of the low-energy states in the daughter nuclides ${ }^{180,182} \mathrm{Pt}$ are very similar [46]. Therefore the difference in $\beta$-decay patterns should be related predominantly to the difference in the structure of the parent ${ }^{180,182} \mathrm{Au}$ isotopes.

\section{Hyperfine structure measurements for ${ }^{180,182} \mathrm{Au}$}

To gain further insight into the possible g.s. spin assignment for ${ }^{180} \mathrm{Au}$, the first hfs measurements for both ${ }^{180,182} \mathrm{Au}$ are presented in this section. The comparison of data extracted

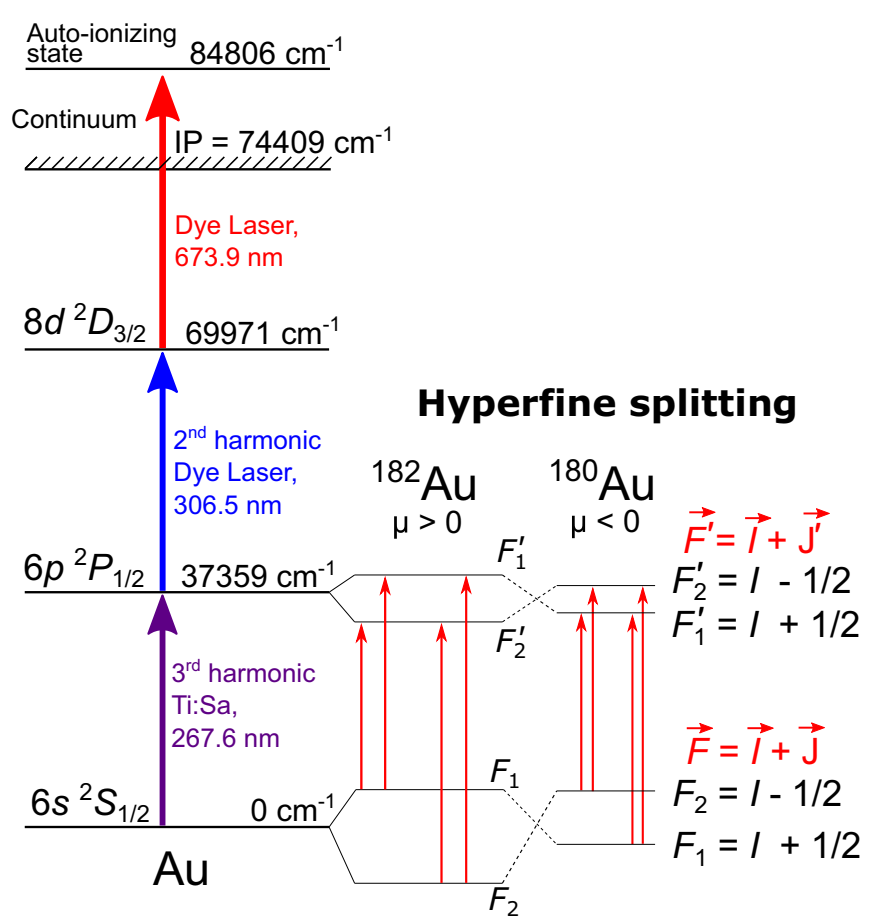

FIG. 6. The three-step resonance photo-ionization scheme used by RILIS to produce ${ }^{180,182} \mathrm{Au}$ ions. The hfs for the 267.6-nm transition for both isotopes is shown (not to scale). $I$ is the nuclear spin, while $J, F$ and $J^{\prime}, F^{\prime}$ are the electron spin and the total angular momenta of the atom for the $6 s^{2} S_{1 / 2}$ g.s. and excited $6 p^{2} P_{1 / 2}$ state, respectively. The four allowed atomic transitions between $F_{1,2}$ and $F_{1,2}^{\prime}$ sublevels are indicated by the colored arrows. Note the inverted positions of the sublevels $F_{1,2}$ and $F_{1,2}^{\prime}$ in ${ }^{180,182} \mathrm{Au}$, which reflects the opposite signs of their respective magnetic moments.

from hfs structure for ${ }^{182} \mathrm{Au}$ with known data (configuration and magnetic dipole moment) for the $I=\left(2^{+}\right)$ground state of ${ }^{182} \mathrm{Au}$, deduced in Refs. [48,49], is instructive for validating the analysis procedures applied for ${ }^{180} \mathrm{Au}$.

\section{Spin assignments for ${ }^{180,182} \mathrm{Au}$}

The left side of Fig. 6 shows the RILIS ionization scheme used to study ${ }^{180,182} \mathrm{Au}$, the same as exploited in our study of ${ }^{177,179} \mathrm{Au}$ in Ref. [15]. The expected hyperfine splitting is shown schematically for the 267.6-nm transition used as the first step in the resonance excitation process. Four transitions between the initial and final states of the hfs structure are possible in both cases, as displayed in the right side of Fig. 6, but their ordering and relative positions depend on the sign and on the value of the magnetic hyperfine constant $a$ (thus on the sign of the magnetic dipole moment).

As discussed below, a positive magnetic dipole moment, $\mu\left({ }^{182} \mathrm{Au}\right)$ derived in Ref. [48] is confirmed by the present data, while the first measurement of $\mu\left({ }^{180} \mathrm{Au}\right)$ and its negative sign were deduced in this work. Figure 6 shows the respective differences in the hfs between the two cases.

The hfs spectra for the 267.6-nm transition were measured by counting photo-ions as a function of the frequency of the first step Ti:Sapphire (Ti:Sa) laser, which was 
used to produce the 267.6-nm beam via a third harmonic generation. The fundamental laser frequency was measured using a wavelength meter (WS7 model by HighFinesse/Angstrom). Further details of the scanning procedure can be found in Refs. [13,15,29]. Two hfs spectra for ${ }^{182} \mathrm{Au}$ were measured by their ion counting with the MR-ToF method, which enables isobaric separation for gold ions from the surface ionized thallium ions by applying time of flight gate. This method was discussed in our recent study of ${ }^{187} \mathrm{Au}$ [17] in the same experimental campaign. Five hfs spectra were measured for ${ }^{180} \mathrm{Au}$ : two with the Windmill $\alpha$-decay counting and three with MR-ToF MS. The $\alpha$-energy range of $5300-5700 \mathrm{keV}$ was used for ${ }^{180} \mathrm{Au}$ in the WM case [cf. Fig. 1(a)]. The spectral resolution is limited by a cumulative effect of the Doppler broadening of atomic lines in the hot cavity of RILIS [13], laser line width, and possible saturation broadening.

An example of an MR-ToF hfs spectrum for ${ }^{182} \mathrm{Au}$ is shown in Fig. 7(a). The four expected transitions are clearly resolved with the FWHM of single resonance equal to $4.8 \mathrm{GHz}$. With $\mu$ $>0$, the two left peaks at lower laser frequencies correspond to transitions $F_{1} \rightarrow F_{1}^{\prime}, F_{2}^{\prime}$, as shown in Fig. 6. The two right peaks at the higher laser frequencies correspond to the two transitions from the $F_{2}$ sublevel (cf. Fig. 6).

The theoretical ratio, $\left(r_{\mathrm{th}}\right)$, of the sum of the intensities for the transitions mentioned above is determined by a simple expression as a function of nuclear spin $I$, see, e.g., the derivation in Ref. [50]:

$$
r_{\mathrm{th}}(I)=\frac{\Gamma\left(F_{1} \rightarrow F_{2}^{\prime}\right)+\Gamma\left(F_{1} \rightarrow F_{1}^{\prime}\right)}{\Gamma\left(F_{2} \rightarrow F_{2}^{\prime}\right)+\Gamma\left(F_{2} \rightarrow F_{1}^{\prime}\right)}=\frac{2 F_{1}+1}{2 F_{2}+1}=\frac{I+1}{I},
$$

where $\Gamma$ is the intensity of the respective transition. This ratio only weakly depends on laser saturation and other factors, which may distort the spectrum shape [51]. For spin assignments of $I=1,2$, and 3 , the theoretical ratio values are $r_{\mathrm{th}}=$ $2,1.5$, and 1.33 , respectively.

Experimentally, this ratio can be calculated by integrating the two broadened left and two broadened right peaks in Fig. 7(a), respectively. The weighted mean of the experimental $r$ values extracted from the two available hfs spectra for ${ }^{182} \mathrm{Au}$, $r_{\exp }=1.61(15)$, further supports the $I\left({ }^{182} \mathrm{Au}\right)=(2)$ spin assignment, proposed earlier in Ref. [49] as most likely, as well as the validity of the $r$-ratio method.

Furthermore, the hfs spectra for ${ }^{182} \mathrm{Au}$ were fitted using Voigt profiles. With the spin assignment of $I\left({ }^{182} \mathrm{Au}\right)=2$, the measured hfs pattern is accurately reproduced by the fit, as shown by the red line in Fig. 7(a), which gives further confirmation on the validity of this spin assignment.

We now turn to the hfs of ${ }^{180} \mathrm{Au}$ shown in Figs. 7(b) and 7(c). As seen in these spectra, the hfs of the upper level in ${ }^{180} \mathrm{Au}$ was not resolved, thus only two peaks have been observed. Due to the negative sign of the magnetic moment (discussed below), the ordering of the $F_{1}, F_{2}$, and $F_{1}^{\prime}, F_{2}^{\prime}$ sublevels is reversed relative to ${ }^{182} \mathrm{Au}$, see Fig. 6 . Therefore, for ${ }^{180} \mathrm{Au}$, the experimental $r$ value is equal to the ratio of the integrals under the right and left peaks in Figs. 7(b) and 7(c), respectively.

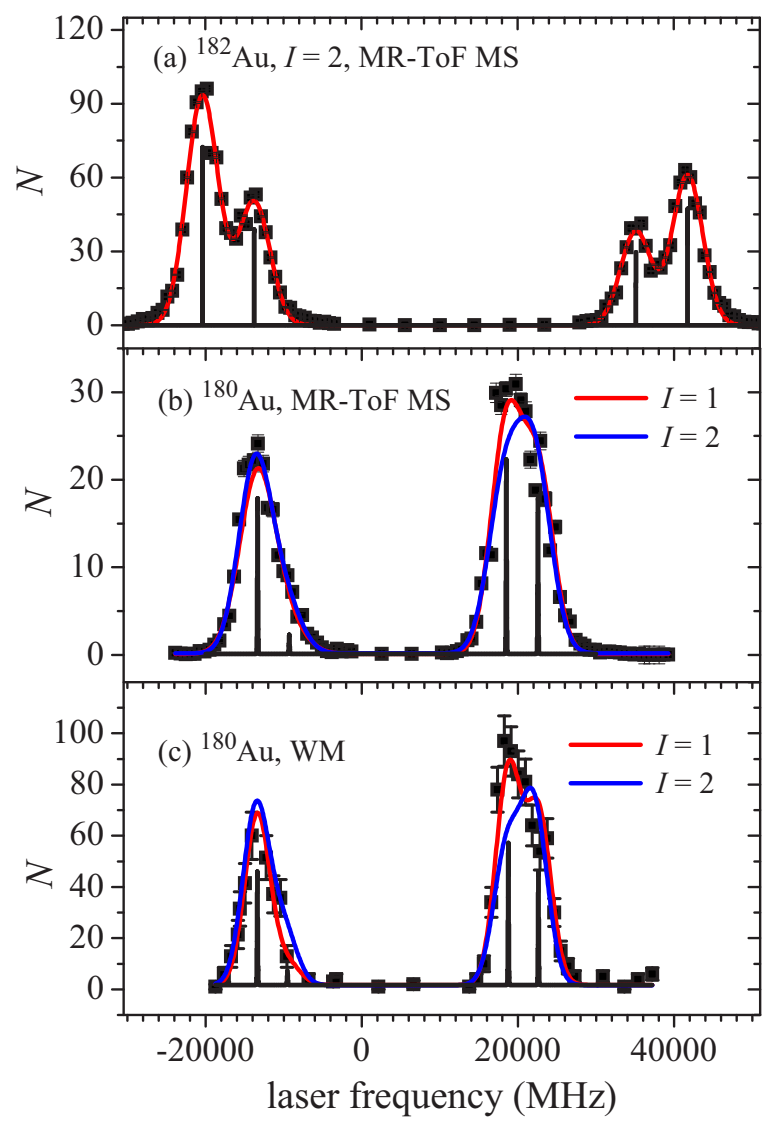

FIG. 7. Examples of the hfs spectra (filled black squares) using the 267.6-nm transition for: (a) ${ }^{182} \mathrm{Au}$ as measured by the MR-ToF MS; (b) and (c) ${ }^{180} \mathrm{Au}$ by using the MR-ToF MS or Windmill decay station, respectively. The zero frequency corresponds to the wave number $37358.9 \mathrm{~cm}^{-1}$. Expected theoretical positions and intensities for the four hfs components, assuming $I=2$ for ${ }^{182} \mathrm{Au}$ and $I=1$ for ${ }^{180} \mathrm{Au}$, are shown by the narrow vertical black lines. The Voigt-profile fits for each experimental hfs spectrum are shown by red lines (spin assignments $I\left({ }^{182} \mathrm{Au}\right)=2$ and $\left.I\left({ }^{180} \mathrm{Au}\right)=1\right)$, and by blue line for $I\left({ }^{180} \mathrm{Au}\right)=2$. Fitting with $I=3$ is not shown on (b) and (c) as it is visually indistinguishable from that of $I=2$.

The weighted mean value, $r_{\exp }=2.06(20)$, for the five hfs spectra available for ${ }^{180} \mathrm{Au}$ indicates a strong preference for an $I=1$ assignment. On the other hand, fits with $I=2,3$ spin assignments, which could be possible from the $\beta$-decay data if there was a direct $\beta$-decay feeding to $4^{+}$states, do not reproduce the details of the shape of the observed spectra, as seen in Figs. 7(b) and 7(c), strengthening the case for an $I=$ 1 value.

To summarize, the hfs analysis for ${ }^{180,182} \mathrm{Au}$ clearly suggests a difference in their spin values, with confirmed $I=(2)$ for ${ }^{182} \mathrm{Au}$ and suggested $I=(1)$ for ${ }^{180} \mathrm{Au}$. These inferences could naturally explain the difference in the relative feeding of the $2_{1}^{+}$and $4_{1}^{+}$states in the respective $\beta$-decay patterns of ${ }^{180,182} \mathrm{Au} \rightarrow{ }^{180,182} \mathrm{Pt}$, see Sec. III B. 
TABLE II. Possible neutron-proton configurations that could couple to produce $I=2$ in ${ }^{182} \mathrm{Au}$ and $I=1$ in ${ }^{180} \mathrm{Au}$. The magnetic dipole moment, $\mu_{\text {calc }}$, of each configuration was calculated using the deformed additivity relations, Eq. (7). The configurations marked in bold correspond to the ground states of ${ }^{180,182} \mathrm{Au}$ as proposed by this work, see the main text. The last two columns show the magnetic moments deduced in this study within the Ekström et al. approach [6] and with the RHFA correction following the prescription from Ref. [16], respectively.

\begin{tabular}{lllrrr}
\hline \hline Isotope & $\pi \otimes v$ configuration & $I^{\pi}$ & $\mu_{\text {calc }}\left(\mu_{N}\right)$ & $\mu_{\exp }\left(\mu_{N}\right),[6]$ & $\mu_{\exp }\left(\mu_{N}\right),[16]$ \\
\hline${ }^{182} \mathrm{Au}$ & $\mathbf{3} / \mathbf{2}^{-}[\mathbf{5 3 2}] \mathbf{h}_{\mathbf{9} / 2} \otimes \mathbf{1} / \mathbf{2}^{-}[\mathbf{5 2 1}] \mathbf{p}_{\mathbf{3} / 2}$ & $2^{+}$ & $1.22(35)$ & $1.53(7)$ & $1.66(9)$ \\
& $3 / 2^{-}[532] h_{9 / 2} \otimes 7 / 2^{+}[633] i_{13 / 2}$ & $2^{-}$ & $-1.09(27)$ & & \\
& $1 / 2^{-}[541] h_{9 / 2} \otimes 5 / 2^{-}[512] h_{9 / 2}$ & $2^{+}$ & $-0.73(20)$ & & $-0.83(9)$ \\
${ }^{180} \mathrm{Au}$ & $\mathbf{3} \mathbf{2}^{-}[\mathbf{5 3 2}] \mathbf{h}_{\mathbf{2} / 2} \otimes \mathbf{5} / \mathbf{2}^{-}[\mathbf{5 1 2}] \mathbf{h}_{\mathbf{2} / 2}$ & $1^{+}$ & $-0.87(21)$ & $-0.74(4)$ & \\
& $7 / 2^{+}[404] g_{7 / 2} \otimes 5 / 2^{-}[512] h_{9 / 2}$ & $1^{-}$ & $2.02(13)$ & & \\
& $1 / 2^{-}[541] h_{9 / 2} \otimes 1 / 2^{-}[521] p_{3 / 2}$ & $1^{+}$ & $0.58(20)$ & & \\
& $5 / 2^{+}[402] d_{5 / 2} \otimes 7 / 2^{-}[514] f_{7 / 2}$ & $1^{-}$ & $-1.51(20)$ & & \\
&
\end{tabular}

\section{Experimental magnetic moments for the ground states of ${ }^{180,182} \mathrm{Au}$}

The positions of the hyperfine components as a function of the scanning laser frequency in Fig. 7 are determined by the expression:

$$
v_{F, F^{\prime}}=v_{0}+a(6 p) \frac{K^{\prime}}{2}-a(6 s) \frac{K}{2}
$$

where $\nu_{0}$ is the centroid frequency of the hfs, the prime symbols denote the upper level of the atomic transition (see definitions in Fig. 6), $K=F(F+1)-I(I+1)-J(J+1)$, and $a(n l)$ is the magnetic hyperfine coupling constant for the atomic level with the quantum numbers $n$ and $l$. The fits of hfs spectra for ${ }^{182} \mathrm{Au}$ by using Voigt profiles [29], with an $I=2$ assumption, resulted in a value of $a\left(6 s,{ }^{182} \mathrm{Au}\right)=22180(80)$ $\mathrm{MHz}$ and a ratio of $\frac{a_{6 p}}{a_{6 s}}\left({ }^{182} \mathrm{Au}\right)=0.119(5)$.

To determine the magnetic dipole moments the standard relation was used:

$$
\mu=\mu_{\mathrm{ref}} \frac{I_{A}}{I_{\mathrm{ref}}} \frac{a_{A}}{a_{\mathrm{ref}}}\left(1+{ }^{\mathrm{ref}} \Delta^{A}\right),
$$

where the subscript (superscript) "ref" denotes a reference isotope $\left({ }^{197} \mathrm{Au}\right)$ with known $\mu$ and $a$ values, and ${ }^{\text {ref }} \Delta^{A}$ is a relative hyperfine anomaly (RHFA) stemming from the nonpoint-like charge and magnetization distribution inside the nucleus, see, e.g., Refs. [6,52] and references therein. In most known nuclei, the RHFA correction is small, of an order of $\left(10^{-4}-10^{-2}\right)$, and is usually omitted in Eq. (5).

However, it is known that in some gold isotopes the RHFA correction is very large, e.g., ${ }^{197} \Delta^{198}=0.0853(8)$ [53]. Therefore, the reliable estimation of the RHFA is necessary in order to obtain magnetic moment values for gold isotopes far from stability.

Usually, the prescription by Ekström et al. [6] is used to account for large RHFA in gold when calculating their magnetic moments:

$$
\mu=\frac{a(6 s) I}{29005} \mu_{N}
$$

However, it has recently been shown in Ref. [16] that this prescription is based on the not well-justified assumptions and should be reconsidered.

As shown in Ref. [16], the RHFA values can be directly deduced from the ratio of the magnetic hfs constants for different atomic states of a nucleus, $6 p$ and $6 s$ in the case of gold. This ratio, $a_{6 p}^{A} / a_{6 s}^{A}$, depends on the atomic mass number $A$, because different atomic states differ in sensitivity to the nuclear magnetization distribution (see details in Ref. [16]). Applying this procedure, we obtain: ${ }^{197} \Delta^{182}=0.17(7)$.

With $a_{6 s}\left({ }^{197} \mathrm{Au}\right)=3049.660092(7) \mathrm{MHz}$ [54], $a_{6 p}\left({ }^{197} \mathrm{Au}\right)$ $=312.7(1.2) \mathrm{MHz}$ [55], and $\mu\left({ }^{197} \mathrm{Au}\right)=0.14574(4) \mu_{N}$ from Ref. [54] with diamagnetic correction from Ref. [56], we obtain by Eq. (5) the magnetic moment for ${ }^{182} \mathrm{Au}$ which includes the RHFA correction: $\mu\left({ }^{182} \mathrm{Au}\right)=1.66(9) \mu_{N}$.

Using the same procedure for ${ }^{180} \mathrm{Au}$, the hfs spectra were fitted with an $I=1$ assumption, resulting in the values of $a_{6 s}\left({ }^{180} \mathrm{Au}\right)=-21370(220) \mathrm{MHz}, a_{6 p}\left({ }^{180} \mathrm{Au}\right) / a_{6 s}\left({ }^{180} \mathrm{Au}\right)=$ $0.122(10)$, and ${ }^{197} \Delta^{180}=0.21(14)$. Correspondingly, Eq. (5) gives: $\mu\left({ }^{180} \mathrm{Au}\right)=-0.83(9) \mu_{N}$. The latter value will be used in Sec. IV A 2 to evaluate the configuration of ${ }^{180} \mathrm{Au}$.

The experimental magnetic moments for ${ }^{180,182} \mathrm{Au}$ deduced with the Ekström et al. prescription [Eq. (6)] and with the approach by Barzakh et al. [Eq. (5)] are shown in Table II. One can notice a difference by $\approx 10 \%$ between the two methods.

\section{DISCUSSION}

\section{A. Derivation of magnetic moments using the deformed additivity relation}

\section{Proton and neutron Nilsson orbitals relevant for ${ }^{180,182} \mathrm{Au}$}

As shown by the analysis of the isotope shift in ${ }^{180,182} \mathrm{Au}$, these nuclei are deformed with a mean-square quadrupole deformation of $\left\langle\beta^{2}\right\rangle^{1 / 2} \approx 0.28$ [57]. Therefore, to assign configurations for the ground states of ${ }^{180,182} \mathrm{Au}$ we will consider the systematics of the lowest deformed single-particle states near $Z=79$ and $N=101,103$. As seen in Fig. 8, several proton and neutron levels are expected to lie close to the Fermi surface for ${ }^{180,182} \mathrm{Au}$ at $\left\langle\beta^{2}\right\rangle^{1 / 2} \approx 0.2-0.3$. It is worth noting that the ground states of the deformed gold isotopes ${ }^{182-186} \mathrm{Au}$ 


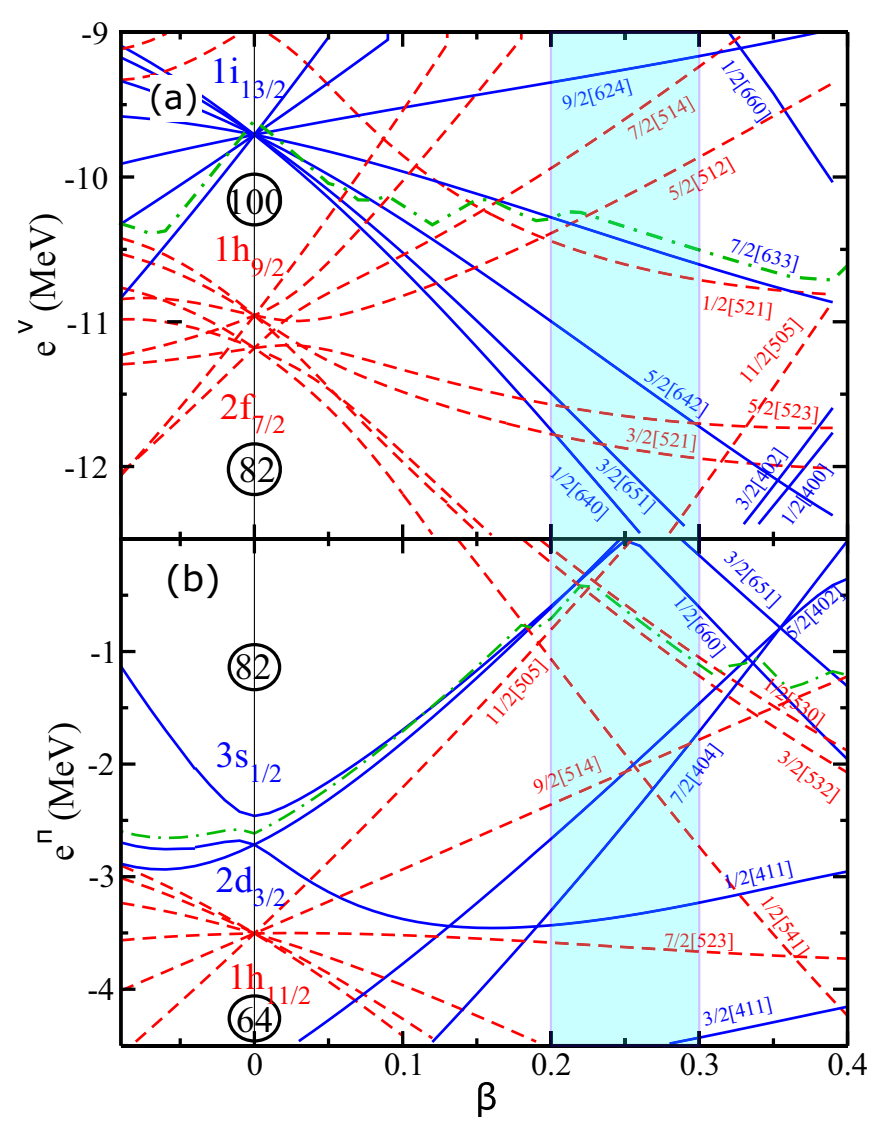

FIG. 8. A Nilsson level diagram relevant to ${ }^{180} \mathrm{Au}$ for (a) neutrons $(82<N<126)$; (b) protons $(50<Z<82)$. The shaded region indicates the region of quadrupole deformation $0.2<\beta<$ 0.3 . The dash-dotted green lines show the positions of respective Fermi surfaces for neutrons and protons.

( $N=103-107)$ are assumed to be based on the $\pi 1 / 2^{-}[541]$ or $\pi 3 / 2^{-}$[532] Nilsson states $[10,11,49,58]$. Note also that the $v 1 / 2^{-}[521]$ orbital is assigned as the ground state configuration in the isotones ${ }^{181} \mathrm{Hg}_{101}$ and ${ }^{179} \mathrm{Pt}_{101}[59,60]$.

The Gallagher-Moszkowski rule [61] may be used to constrain the orbitals near the Fermi surface, which can couple to $I=1$ for ${ }^{180} \mathrm{Au}$ and $I=2$ for ${ }^{182} \mathrm{Au}$. The possible configurations are shown in Table II, where both negative and positive parity states are shown for the sake of completeness. The choice of the most likely configurations for ${ }^{180,182} \mathrm{Au}$ is determined by comparing their experimental $\mu$ values, with the theoretical values for the odd-odd configurations from Table II, calculated using the following additivity expression [62]:

$$
\begin{aligned}
\mu_{\text {calc }} & =\frac{K}{K+1}\left[ \pm g_{K P} \cdot K_{p} \pm g_{K n} \cdot K_{n}+g_{R}\right] \\
K & =\left|K_{p} \pm K_{n}\right|
\end{aligned}
$$

where $K_{p, n}$ is the projection of the angular momentum on the symmetry axis for proton and neutron states, $g_{K}$ is the intrinsic $g$ factor, $g_{R}$ is the rotational gyromagnetic ratio $\left(g_{R}=\right.$ $Z / A \approx 0.4$ is usually adopted for odd-odd nuclei in this region [62]). Signs in Eq. (7) are chosen in accordance with those in Eq. (8). The intrinsic $g_{K}$ factors for the chosen orbitals were calculated by the standard Nilsson-model approach with the different parameters sets [24,63-65], and extracted, when possible, from the measured magnetic moments [66] by the relation [62]:

$$
g_{K} \cdot K=\frac{K+1}{K} \mu_{\text {odd, exp }}-g_{R} .
$$

The uncertainties in the $g_{K}$ values were estimated from the dispersion of the results obtained by the different approaches. The deduced $\mu_{\text {calc }}$ values are shown in Table II.

The previously reported value of $\mu\left({ }^{182} \mathrm{Au}\right)$ originates from $g$-factor measurements employing time-resolved and timeintegral on-line nuclear orientation methods at the NICOLEISOLDE facility, and considering a range of spin assumptions $I=1-5$, see Table 3 of Ref. [48]. By comparing the $g$ factors from the two methods, the authors of Ref. [48] derived $\mu\left({ }^{182} \mathrm{Au}\right)=1.65(15) \mu_{N}$ by assuming $I=3$, which gave the best agreement between the two different experimental techniques, although a spin value of $I=2$ could not be excluded, as was stated by the authors themselves. A subsequent $\beta^{+} / E C$ decay study of ${ }^{182} \mathrm{Hg} \rightarrow{ }^{182} \mathrm{Au}$ at ISOLDE [49] proposed a g.s. spin $I\left({ }^{182} \mathrm{Au}\right)=\left(2^{+}\right)$based on $\gamma-\gamma$ and $e^{-}-\gamma$ coincidence measurements, with the $\pi h_{9 / 2} \otimes$ $v 1 / 2^{-}[521]$ as most probable configuration from evaluating the lowest-energy configurations and systematics from the neighboring ${ }^{184,186} \mathrm{Au}$ isotopes. The $I=\left(2^{+}\right)$value is used in the present NNDC evaluations [24], which reported a value of $\mu\left({ }^{182} \mathrm{Au}\right)=1.30(10) \mu_{N}$ calculated from the average $g=$ $0.65(5)$ value of the two $I=2 g$-factor derivations from Table 3 in Ref. [48]. A $\approx 3 \sigma$ discrepancy between the evaluated value and our result $\left[\mu\left({ }^{182} \mathrm{Au}\right)=1.66(9) \mu_{N}\right]$ may be explained by the fact that different approaches have been used for the hyperfine anomaly estimation in Ref. [48] and in the present study, which follows the method presented in Ref. [16].

Out of three possible configurations, shown in Table II, only the $\mu_{\text {calc }}=1.22(35) \mu_{N}$ is reasonably close to the experimentally deduced value, which further strengthens the choice of $I=2^{+}$and the $3 / 2^{-}[532] h_{9 / 2} \otimes 1 / 2^{-}[521] p_{3 / 2}$ configuration for the ground state of ${ }^{182} \mathrm{Au}$.

\section{Configuration of the $I=\left(1^{+}\right)$ground state in ${ }^{180} \mathrm{Au}$}

As seen from Table II, a reasonable agreement between the experimental value, $\mu_{\exp }\left({ }^{180} \mathrm{Au}\right)=-0.83(9) \mu_{N}$ and the calculated additivity values can only be obtained for the state with $I^{\pi}\left({ }^{180} \mathrm{Au}^{\mathrm{gs}}\right)=1^{+}$, with a $\pi 3 / 2^{-}[532] \otimes v 5 / 2^{-}[512]$ configuration. Thus, the proton state in ${ }^{180} \mathrm{Au}^{\mathrm{gs}}$ is expected to be the same as in ${ }^{184,182} \mathrm{Au}\left(\pi 3 / 2^{-}\right.$[532]), whereas the assigned neutron orbital $\left(v 5 / 2^{-}[512]\right)$ is the same as the ground states of several $N=99$ and 103 nuclei $\left({ }^{177} \mathrm{Pt}_{99}\right.$, ${ }^{175} \mathrm{Os}_{99},{ }^{173} \mathrm{~W}_{99},{ }^{171} \mathrm{Er}_{103},{ }^{173} \mathrm{Yb}_{103},{ }^{175} \mathrm{Hf}_{103}$, see Ref. [65] and the references therein).

\section{B. $\alpha$-decay hindrance factors for ${ }^{180} \mathrm{Au}$}

$\alpha$-decay hindrance factors $\left(\mathrm{HF}_{\alpha}\right)$ are a sensitive probe for providing information on the spin and/or configuration of states linked by the decay, if the properties of either the 
parent or daughter states are well established. In the case of ${ }^{180} \mathrm{Au}$, one could try to use the $\mathrm{HF}_{\alpha}$ values in the decay chain of ${ }^{184} \mathrm{Tl} \rightarrow{ }^{180} \mathrm{Au} \rightarrow{ }^{176} \mathrm{Ir}$. However, the application of this method is not possible in this case for the following reasons. First, as shown below, the tentative 5675/5686-keV $\alpha$ decays are strongly hindered by a factor of $\mathrm{HF}_{\alpha}>1700$. This fact provides an evidence for a large spin and configuration difference between the ground states of ${ }^{180} \mathrm{Au}$ and ${ }^{176} \mathrm{Ir}$, possibly suggesting the validity of the $I\left({ }^{176} \mathrm{Ir}\right)=\left(5^{+}\right)$g.s. assignment made in study [21]. However, no unambiguous conclusions can be drawn at present, especially considering the multitude of low-energy states proposed by other studies $[22,23,25]$. Similarly, there are no direct $\alpha$ decays linking the three known $\alpha$-decaying states with $I=10^{-}, 7^{+}$, and $2^{-}$of ${ }^{184} \mathrm{Tl}$ to the proposed $I=\left(1^{+}\right)$g.s. of ${ }^{180} \mathrm{Au}$ (see Figs. 12 and 13 of Ref. [67]).

That is why we now turn to the discussion of the relative strengths of the f.s. $\alpha$ decays from ${ }^{180} \mathrm{Au}$, which could help to establish the similarities or differences of respective daughter states in ${ }^{176} \mathrm{Ir}$. Figure 2 shows the $\delta_{\alpha}^{2}$ values of each f.s. decay of ${ }^{180} \mathrm{Au}$, calculated using the Rasmussen approach [68] and assuming $\Delta L=0$ decays. The absolute hindrance factor values can be calculated by comparing the $\delta_{\alpha}^{2}$ values for $\alpha$ decays from ${ }^{180} \mathrm{Au}$, to unhindered $\alpha$ decays in the neighboring odd- $A$ nuclei ${ }^{181} \mathrm{Au}$ and ${ }^{179} \mathrm{Au}$.

Based on the data from Ref. [24], values of $\delta_{\alpha}^{2}\left[{ }^{181} \mathrm{Au}\right.$, $\left.5479 \mathrm{keV},\left(3 / 2^{-}\right) \rightarrow 3 / 2^{-}\right]=75(11) \mathrm{keV}$ and $\delta_{\alpha}^{2}\left({ }^{179} \mathrm{Au}\right.$, $\left.5848 \mathrm{keV}, 1 / 2^{+} \rightarrow 1 / 2^{+}\right)=57(4) \mathrm{keV}$ were deduced for these unhindered $\alpha$ decays. The average of these values, $\delta_{\alpha}^{2}=66(6) \mathrm{keV}$, was used as a reference for ${ }^{180} \mathrm{Au}$ in the following analysis. The $5485-\mathrm{keV} \alpha$ decay in ${ }^{180} \mathrm{Au}$ is only weakly hindered with $\mathrm{HF}_{\alpha}=2.8(7)$. Based on this relatively low hindrance, we suggest that the state at $\Delta+195.7 \mathrm{keV}$ in ${ }^{176} \mathrm{Ir}$ fed by the $5485-\mathrm{keV} \alpha$ decay has most likely the same spin $\left(1^{+}\right)$and configuration as the parent ${ }^{180} \mathrm{Au}$ ground state. Further tentative spin assignments could be tried for some of the states linked to the $\Delta+195.7 \mathrm{keV}$ state via $\gamma$ decays with deduced $M 1$ multipolarities (e.g., the 36.5-, 41.5-, and 118-keV transitions). However, we prefer to refrain from such an analysis due to the large number of spin values obtained by coupling several not well-defined angular momenta.

It is interesting to note that, e.g., the 5639-keV and 5598$\mathrm{keV}$ f.s. decays have similar hindrance factors $(\approx 16-18)$, thus hindered rather weakly by $\approx 4$ times relative to the $5485-\mathrm{keV}$ $\alpha$ decay. This may indicate a similarity and/or a possible mixing of the underlying configurations of the states at 36.5, 78.0, and $195.7 \mathrm{keV}$.

As another explanation for relatively low and comparable hindrance factors for several f.s. $\alpha$ decays in ${ }^{180} \mathrm{Au}$, one could consider a possibility of decays to excited states of the same rotational band in the (deformed) daughter. There are such cases known in the literature, including in the region close to ${ }^{180} \mathrm{Au}$. For example, in the ${ }^{181} \mathrm{Hg} \rightarrow{ }^{177} \mathrm{Pt} \alpha$ decay, the $1 / 2^{-} \rightarrow 1 / 2^{-}$decay is unhindered $\left[\mathrm{HF}_{\alpha}=0.86(12)\right]$, while the two decays to the $\left(3 / 2^{-}\right)$and $5 / 2^{-}$excited states have moderate hindrance factors of 6.5(20) and 7(3), respectively. The $\left(3 / 2^{-}\right)$and $5 / 2^{-}$states are most probably the members of a rotational band built on the $147-\mathrm{keV} 1 / 2^{-}$state fed by unhindered $\alpha$ decay of ${ }^{181} \mathrm{Hg}$. Thus, medium values of $\mathrm{HF}_{\alpha}=5-20$ in this region may be due to the decay to rotational states in the daughter nuclide based on the same configuration as in the parent nucleus. However, at present, it is difficult to assess the validity of this scenario for ${ }^{180} \mathrm{Au}$, because not much is known about the detailed structure of the daughter nucleus ${ }^{176} \mathrm{Ir}$. A dedicated study of both its ground state and of the expected complex pattern of low-lying excited states, as suggested by calculations in Ref. [23], would be needed to shed more light on this scenario.

This additional information could possibly help understand the puzzling fact of the strong hindrance of the tentative 5675$\mathrm{keV} \alpha$ decay relative to the 5485-keV, 5598-keV, and 5539$\mathrm{keV}$ decays. Indeed, an $M 1$ character of the three $\gamma$ transitions at $36.5-\mathrm{keV}, 41.5-\mathrm{keV}$, and $118-\mathrm{keV}$ linking the four states fed by these decays suggests that the spin of the state at $\Delta$ fed by the $5675 \mathrm{keV}$ decay should be not too much distinctive from the states fed by other $\alpha$ decays. Therefore, the $\alpha$ decay hindrance for the $5675-\mathrm{keV}$ decay should be much lower than observed. The presence of the direct, albeit rather weak, 195.7-keV parity-conserving transition following the unhindered 5485-keV decay also highlights this discrepancy.

A similar argument cannot, however, yet be applied for the case of the $205.2 \mathrm{keV}$ transition, as the parity of the lowest state in ${ }^{176} \mathrm{Ir}$ is not known. For example, a negative parity of this state (thus, an $E 1$ or $M 2$ multipolarity for the $205.2 \mathrm{keV}$ decay) would indeed cause a strong hindrance for the $5686-\mathrm{keV}$ decay. This is both due to the very strong sensitivity of the $\alpha$-decay process to the parity change and a need for a respective substantial change of the underlying configuration of the state.

\section{CONCLUSION}

A detailed nuclear spectroscopy study of ${ }^{180} \mathrm{Au}$ was performed using the RILIS, Windmill decay station and MR-ToF MS setups at ISOLDE. Due to the pure and intense beam provided by the laser ionization coupled to mass separation, complex fine-structure $\alpha$ decays have been identified and their properties, such as the relative intensities and $\mathrm{HF}_{\alpha}$ values, were deduced.

The first measurements of the hfs for atomic transitions in ${ }^{180,182} \mathrm{Au}$ were also presented, from which the respective magnetic dipole moments have been extracted. A spin assignment of $I^{\pi}=\left(1^{+}\right)$is proposed for the ground state of ${ }^{180} \mathrm{Au}$, with a $\left(\pi 3 / 2^{-}[532] \otimes \nu 5 / 2^{-}[512]\right)$ configuration. The complementary hfs data for ${ }^{182} \mathrm{Au}$ were also obtained, which supports the earlier ground-state spin assignment of $I^{\pi}$ $=\left(2^{+}\right)$and proposes its $\pi 3 / 2^{-}[532] h_{9 / 2} \otimes v 1 / 2^{-}[521] p_{3 / 2}$ configuration.

\section{ACKNOWLEDGMENTS}

We would like to acknowledge the support of the ISOLDE Collaboration and technical teams. We thank the GSI target group for supplying the thin carbon foils used in WM measurements. This work has received funding from the European Union Horizon 2020 research and innovation programme 
under Grant Agreement No. 654002, and also funding through the European Union Seventh Framework Programme for Research and Technological Development under Grant Agreements No. 262010 (ENSAR), No. 267194 (COFUND), and No. 289191 (LA3NET). The work was further supported by RFBR according to the Research Project No. 19-0200005, by the German Federal Ministry of Education and Research (BMBF, Grants No. 05P12HGCI1, No. 05P15HGCIA,
No. 05P18HGCIA), by grants from the U.K. Science and Technology Facilities Council, by FWO-Vlaanderen (Belgium), by GOA/2010/010 (BOF KU Leuven), by the Interuniversity Attraction Poles Programme initiated by the Belgian Science Policy Office (BriX network P7/12), by French IN2P3, by the Slovak Research and Development Agency (Contract No. APVV-14-0524) and the Slovak Grant Agency VEGA (Contract No. 1/0532/17).
[1] A. N. Andreyev, M. Huyse, P. Van Duppen, L. Weissman, D. Ackermann, J. Gerl, F. P. Heßberger, S. Hofmann, A. Kleinböhl, G. Münzenberg, S. Reshitko, C. Schlegel, H. Schaffner, P. Cagarda, M. Matos, S. Saro, A. Keenan, C. Moore, C. D. O'Leary, R. D. Page, M. Taylor, H. Kettunen, M. Leino, A. Lavrentiev, R. Wyss, and K. Heyde, Nature (London) 405, 430 (2000).

[2] K. Heyde and J. L. Wood, Rev. Mod. Phys. 83, 1467 (2011).

[3] T. Otsuka and Y. Tsunoda, J. Phys. G: Nucl. Part. Phys. 43, 024009 (2016).

[4] J. L. Wood and K. Heyde, J. Phys. G: Nucl. Part. Phys. 43, 020402 (2016).

[5] B. A. Marsh, T. Day Goodacre, S. Sels, Y. Tsunoda, B. Andel, A. N. Andreyev, N. A. Althubiti, D. Atanasov, A. E. Barzakh, J. Billowes, K. Blaum, T. E. Cocolios, J. G. Cubiss, J. Dobaczewski, G. J. Farooq-Smith, D. V. Fedorov, V. N. Fedosseev, K. T. Flanagan, L. P. Gaffney, L. Ghys, M. Huyse, S. Kreim, D. Lunney, K. M. Lynch, V. Manea, Y. Martinez Palenzuela, P. L. Molkanov, T. Otsuka, A. Pastore, M. Rosenbusch, R. E. Rossel, S. Rothe, L. Schweikhard, M. D. Seliverstov, P. Spagnoletti, C. Van Beveren, P. Van Duppen, M. Veinhard, E. Verstraelen, A. Welker, K. Wendt, F. Wienholtz, R. N. Wolf, A. Zadvornaya, and K. Zuber, Nature Phys. 14, 1163 (2018).

[6] C. Ekström, L. Robertsson, S. Ingelman, G. Wannberg, and I. Ragnarsson, Nucl. Phys. A 348, 25 (1980).

[7] K. Wallmeroth, G. Bollen, A. Dohn, P. Egelhof, J. Grüner, F. Lindenlauf, U. Krönert, J. Campos, A. Rodriguez Yunta, M. J. G. Borge, A. Venugopalan, J. L. Wood, R. B. Moore, and H.-J. Kluge, Phys. Rev. Lett. 58, 1516 (1987).

[8] U. Krönert, S. Becker, G. Bollen, M. Gerber, T. Hilberath, H.-J. Kluge, and G. Passler, Z. Phys. A 331, 521 (1988).

[9] K. Wallmeroth, G. Bollen, A. Dohn, P. Egelhof, U. Krönert, M. J. G. Borge, J. Campos, A. Rodriguez Yunta, K. Heyde, C. De Coster, J. L. Wood, and H.-J. Kluge, Nucl. Phys. A 493, 224 (1989).

[10] F. Le Blanc, J. Obert, J. Oms, J. C. Putaux, B. Roussière, J. Sauvage, J. Pinard, L. Cabaret, H. T. Duong, G. Huber, M. Krieg, V. Sebastian, J. Crawford, J. K. P. Lee, J. Genevey, and F. Ibrahim (ISOLDE Collaboration), Phys. Rev. Lett. 79, 2213 (1997).

[11] G. Savard, J. E. Crawford, J. K. Lee, G. Thekkadath, H. T. Duong, J. Pinard, F. Le Blanc, P. Kilcher, J. Obert, J. Oms, J. C. Putaux, B. Roussiere, and J. Sauvage, Nucl. Phys. A 512, 241 (1990).

[12] J. Sauvage, F. Ibrahim, B. Roussière, J. Genevey, A. Gizon, G. Marguier, P. Kilcher, A. Knipper, R. Béraud, G. Căta-Danil, F. Le Blanc, J. Obert, J. Oms, J. C. Putaux, C. Richard-Serre,
A. Wojtasiewicz, the ISOLDE, and NICOLE-Collaborations, Eur. Phys. A 25, 5 (2005).

[13] B. Marsh, B. Andel, A. N. Andreyev, S. Antalic, D. Atanasov, A. Barzakh, B. Bastin, C. Borgmann, L. Capponi, T. Cocolios, T. Day Goodacre, M. Dehairs, X. Derkx, H. De Witte, D. Fedorov, V. Fedosseev, G. Focker, D. Fink, K. Flanagan, S. Franchoo, L. Ghys, M. Huyse, N. Imai, Z. Kalaninova, U. Köster, S. Kreim, N. Kesteloot, Y. Kudryavtsev, J. Lane, N. Lecesne, V. Liberati, D. Lunney, K. Lynch, V. Manea, P. Molkanov, T. Nicol, D. Pauwels, L. Popescu, D. Radulov, E. Rapisarda, M. Rosenbusch, R. Rossel, S. Rothe, L. Schweikhard, M. Seliverstov, S. Sels, A. Sjödin, V. Truesdale, C. Van Beveren, P. Van Duppen, K. Wendt, F. Wienholtz, R. Wolf, and S. G. Zemlyanoy, Nucl. Instrum. Methods B 317, 550 (2013).

[14] R. Catherall, W. Andreazza, M. Breitenfeldt, A. Dorsival, G. J. Focker, T. P. Gharsa, G. T. J, J.-L. Grenard, F. Locci, P. Martins, S. Marzari, J. Schipper, A. Shornikov, and T. Stora, J. Phys. G: Nucl. Part. Phys. 44, 094002 (2017).

[15] J. G. Cubiss, A. E. Barzakh, A. N. Andreyev, M. Al Monthery, N. Althubiti, B. Andel, S. Antalic, D. Atanasov, K. Blaum, T. E. Cocolios, T. Day Goodacre, R. P. de Groote, A. De Roubin, G. J. Farooq-Smith, D. V. Fedorov, V. N. Fedosseev, R. Ferrer, D. A. Fink, L. P. Gaffney, L. Ghys, A. Gredley, R. D. Harding, F. Herfurth, M. Huyse, N. Imai, D. T. Joss, U. Köster, S. Kreim, V. Liberati, D. Lunney, K. M. Lynch, V. Manea, B. A. Marsh, Y. Martinez Palenzuela, P. L. Molkanov, P. Mosat, D. Neidherr, G. G. O’Neill, R. D. Page, T. J. Procter, E. Rapisarda, M. Rosenbusch, S. Rothe, K. Sandhu, L. Schweikhard, M. D. Seliverstov, S. Sels, P. Spagnoletti, V. L. Truesdale, C. Van Beveren, P. Van Duppen, M. Veinhard, M. Venhart, M. Veselský, F. Wearing, A. Welker, F. Wienholtz, R. N. Wolf, S. G. Zemlyanoy, and K. Zuber, Phys. Lett. B 786, 355 (2018).

[16] A. E. Barzakh, D. Atanasov, A. N. Andreyev, M. Al Monthery, N. A. Althubiti, B. Andel, S. Antalic, K. Blaum, T. E. Cocolios, J. G. Cubiss, P. Van Duppen, T. D. Goodacre, A. de Roubin, Y. A. Demidov, G. J. Farooq-Smith, D. V. Fedorov, V. N. Fedosseev, D. A. Fink, L. P. Gaffney, L. Ghys, R. D. Harding, D. T. Joss, F. Herfurth, M. Huyse, N. Imai, M. G. Kozlov, S. Kreim, D. Lunney, K. M. Lynch, V. Manea, B. A. Marsh, Y. Martinez Palenzuela, P. L. Molkanov, D. Neidherr, R. D. Page, M. Rosenbusch, R. E. Rossel, S. Rothe, L. Schweikhard, M. D. Seliverstov, S. Sels, C. Van Beveren, E. Verstraelen, A. Welker, F. Wienholtz, R. N. Wolf, and K. Zuber, Phys. Rev. C 101, 034308 (2020).

[17] A. E. Barzakh, D. Atanasov, A. N. Andreyev, M. Al Monthery, N. A. Althubiti, B. Andel, S. Antalic, K. Blaum, T. E. Cocolios, J. G. Cubiss, P. Van Duppen, T. D. Goodacre, A. de Roubin, 
Y. A. Demidov, G. J. Farooq-Smith, D. V. Fedorov, V. N. Fedosseev, D. A. Fink, L. P. Gaffney, L. Ghys, R. D. Harding, D. T. Joss, F. Herfurth, M. Huyse, N. Imai, M. G. Kozlov, S. Kreim, D. Lunney, K. M. Lynch, V. Manea, B. A. Marsh, Y. Martinez Palenzuela, P. L. Molkanov, D. Neidherr, R. D. Page, M. Rosenbusch, R. E. Rossel, S. Rothe, L. Schweikhard, M. D. Seliverstov, S. Sels, C. Van Beveren, E. Verstraelen, A. Welker, F. Wienholtz, R. N. Wolf, and K. Zuber, Phys. Rev. C 101, 064321 (2020).

[18] J. L. Wood, E. E. Zganjar, C. D. Coster, and K. Heyde, Nucl. Phys. A 651, 323 (1999).

[19] J. G. Keller, K.-H. Schmidt, F. P. Heßberger, G. Münzenberg, W. Reisdorf, H.-G. Clerc, and C.-C. Sahm, Nucl. Phys. A 452, 173 (1986).

[20] J. Wauters, P. Dendooven, M. Huyse, G. Reusen, P. Van Duppen, R. Kirchner, O. Klepper, and E. Roeckl, Z. Phys. A 345, 21 (1993).

[21] U. Bosch, P. Koschel, W.-D. Schmidt-Ott, V. Freystein, T. Hild, F. Meissner, H. Salewski, U. Ellmers, and R. Michaelsen, Z. Phys. A 336, 359 (1990).

[22] T. Kibédi, G. D. Dracoulis, A. P. Byrne, and P. M. Davidson, Nucl. Phys. A 567, 183 (1994).

[23] W. Hua-Lei, Z. Yu-Hu, Z. Xiao-Hong, G. Ying-Xiang, L. Xiang-Guo, X. Shu-Wei, X. Yuan-Xiang, L. Min-Liang, Z. Yong, X. Ye-Bing, X. Cheng-Ying, S. Li-Tao, L. Peng, Y. Hai-Ping, and G. Wen-Tao, Chin. Phys. Lett. 22, 2211 (2005).

[24] BNL, NNDC, Evaluated Nuclear Structure Data File Search and Retrieval (2019).

[25] R. A. Bark, A. M. Baxter, A. P. Byrne, P. M. Davidson, G. D. Dracoulis, S. M. Mullins, T. R. McGoram, and R. T. Newman, Phys. Rev. C 67, 014320 (2003).

[26] V. Fedosseev, K. Chrysalidis, T. D. Goodacre, B. Marsh, S. Rothe, C. Seiffert, and K. Wendt, J. Phys. G: Nucl. Part. Phys. 44, 084006 (2017).

[27] L. Ghys, A. N. Andreyev, M. Huyse, P. Van Duppen, S. Antalic, A. Barzakh, L. Capponi, T. E. Cocolios, J. Cubiss, X. Derkx, H. De Witte, J. Elseviers, F. P. Heßberger, Z. Kalaninová, U. Köster, J. F. W. Lane, V. Liberati, S. Mitsuoka, Y. Nagame, K. Nishio, S. Ota, D. Pauwels, R. D. Page, L. Popescu, D. Radulov, M. M. Rajabali, E. Rapisarda, K. Sandhu, V. L. Truesdale, P. Van den Bergh, and Y. Wakabayashi, Phys. Rev. C 100, 054310 (2019).

[28] R. N. Wolf, F. Wienholtz, D. Atanasov, D. Beck, K. Blaum, C. Borgmann, F. Herfurth, M. Kowalska, S. Kreim, Y. A. Litvinov, D. Lunney, V. Manea, D. Neidherr, M. Rosenbusch, L. Schweikhard, J. Stanja, and K. Zuber, Int. J. Mass. Spectrom. 349-350, 123 (2013).

[29] J. G. Cubiss, A. E. Barzakh, M. D. Seliverstov, A. N. Andreyev, B. Andel, S. Antalic, P. Ascher, D. Atanasov, D. Beck, J. Bieroń, K. Blaum, C. Borgmann, M. Breitenfeldt, L. Capponi, T. E. Cocolios, T. Day Goodacre, X. Derkx, H. De Witte, J. Elseviers, D. V. Fedorov, V. N. Fedosseev, S. Fritzsche, L. P. Gaffney, S. George, L. Ghys, F. P. Heßberger, M. Huyse, N. Imai, Z. Kalaninová, D. Kisler, U. Köster, M. Kowalska, S. Kreim, J. F. W. Lane, V. Liberati, D. Lunney, K. M. Lynch, V. Manea, B. A. Marsh, S. Mitsuoka, P. L. Molkanov, Y. Nagame, D. Neidherr, K. Nishio, S. Ota, D. Pauwels, L. Popescu, D. Radulov, E. Rapisarda, J. P. Revill, M. Rosenbusch, R. E. Rossel, S. Rothe, K. Sandhu, L. Schweikhard, S. Sels, V. L. Truesdale, C. Van Beveren, P. Van den Bergh, Y. Wakabayashi,
P. Van Duppen, K. D. A. Wendt, F. Wienholtz, B. W. Whitmore, G. L. Wilson, R. N. Wolf, and K. Zuber, Phys. Rev. C 97, 054327 (2018).

[30] J. P. Husson, C. F. Liang, and C. Richard-Serre, J. Physique Lett. 38, 245 (1977).

[31] K. M. Lynch, T. E. Cocolios, N. Althubiti, G. J. Farooq-Smith, W. Gins, and A. J. Smith, Nucl. Instrum. Meth. A 844, 14 (2017).

[32] A. Rytz, At. Data Nucl. Data Tables 47, 205 (1991).

[33] A. Siivola, Nucl. Phys. A 109, 231 (1968).

[34] J. G. Cubiss, R. D. Harding, A. N. Andreyev, N. Althubiti, B. Andel, S. Antalic, A. E. Barzakh, T. E. Cocolios, T. D. Goodacre, G. J. Farooq-Smith, D. V. Fedorov, V. N. Fedosseev, L. P. Gaffney, L. Ghys, M. Huyse, K. M. Lynch, B. A. Marsh, Y. M. Palenzuela, P. L. Molkanov, R. E. Rossel, S. Rothe, M. D. Seliverstov, S. Sels, P. Spagnoletti, C. Van Beveren, P. Van Duppen, M. Veinhard, E. Verstraelen, and A. Zadvornaya, Phys. Rev. C 101, 014314 (2020).

[35] A. Siilvola, Nucl. Phys. 84, 385 (1966).

[36] F. G. Kondev, R. V. Janssens, M. P. Carpenter, K. Abu Saleem, I. Ahmad, M. Alcorta, H. Amro, P. Bhattacharyya, L. T. Brown, J. Caggiano, C. N. Davids, S. M. Fischer, A. Heinz, B. Herskind, R. A. Kaye, T. L. Khoo, T. Lauritsen, C. J. Lister, W. C. Ma, R. Nouicer, J. Ressler, W. Reviol, L. L. Riedinger, D. G. Sarantites, D. Seweryniak, S. Siem, A. A. Sonzogni, J. Uusitalo, P. G. Varmette, and I. Wiedenhöver, Phys. Rev. C 62, 044305 (2000).

[37] V. Manea, P. Ascher, D. Atanasov, A. E. Barzakh, D. Beck, K. Blaum, C. Borgmann, M. Breitenfeldt, R. B. Cakirli, T. E. Cocolios, T. Day Goodacre, D. V. Fedorov, V. N. Fedosseev, S. George, F. Herfurth, M. Kowalska, S. Kreim, Y. A. Litvinov, D. Lunney, B. Marsh, D. Neidherr, M. Rosenbusch, R. E. Rossel, S. Rothe, L. Schweikhard, F. Wienholtz, R. N. Wolf, and K. Zuber, Phys. Rev. C 95, 054322 (2017).

[38] Y. Litvinov, H. Geissel, T. Radon, F. Attallah, G. Audi, K. Beckert, F. Bosch, M. Falch, B. Franzke, M. Hausmann, M. Hellström, T. Kerscher, O. Klepper, H.-J. Kluge, C. Kozhuharov, K. Löbner, G. Münzenberg, F. Nolden, Y. Novikov, W. Quint, Z. Patyk, H. Reich, C. Scheidenberger, B. Schlitt, M. Steck, K. Sümmerer, L. Vermeeren, M. Winkler, T. Winkler, and H. Wollnik, Nucl. Phys. A 756, 3 (2005).

[39] T. Kibédi, T. W. Burrows, M. B. Trzhaskovskaya, P. M. Davidson, and C. W. Nestor, Nucl. Instrum. Meth. A 589, 202 (2008)

[40] S. Agostinelli et al., Nucl. Instrum. Meth. A 506, 250 (2003).

[41] J. Allison et al., Nucl. Instrum. Meth. A 835, 186 (2016).

[42] F. P. Heßberger, S. Hofmann, G. Münzenberg, K.-H. Schmidt, P. Armbruster, and R. Hingmann, Nucl. Instrum. Meth. A 274, 522 (1989).

[43] J. Elseviers, A. N. Andreyev, S. Antalic, A. Barzakh, N. Bree, T. E. Cocolios, V. F. Comas, J. Diriken, D. Fedorov, V. N. Fedosseyev, S. Franchoo, J. A. Heredia, M. Huyse, O. Ivanov, U. Köster, B. A. Marsh, R. D. Page, N. Patronis, M. Seliverstov, I. Tsekhanovich, P. Van Den Bergh, J. Van De Walle, P. Van Duppen, M. Venhart, S. Vermote, M. Veselský, and C. Wagemans, Phys. Rev. C 84, 034307 (2011).

[44] R. D. Harding, Ph.D. thesis, University of York, 2020, http:// etheses.whiterose.ac.uk/26900/.

[45] A. Siivola, Nucl. Phys. A 92, 475 (1967).

[46] P. M. Davidson, G. D. Dracoulis, T. Kibédi, A. P. Byrne, S. S. Anderssen, A. M. Baxter, B. Fabricius, G. J. Lane, and A. E. Stuchbery, Nucl. Phys. A 657, 219 (1999). 
[47] M. Wang, G. Audi, F. G. Kondev, W. Huang, S. Naimi, and X. Xu, Chin. Phys. C 41, 030003 (2017).

[48] I. Romanski, I. Berkes, D. E. Brown, M. De Jesus, R. Eder, I. S. Grant, E. Hagn, P. Harding, P. Herzog, B. Hinfurtner, B. Kastelein, H. Postma, J. Prinz, P. Richards, K. Schlösser, N. J. Stone, L. Vanneste, and E. Zech (ISOLDE and NICOLECollaborations), Hyperfine Interact. 75, 457 (1992).

[49] F. Ibrahim, J. Genevey, E. Cottereau, A. Gizon, A. Knipper, F. L. Blanc, G. Marguier, J. Obert, J. Oms, C. Putaux, B. Roussière, J. Sauvage, A. Wojtasiewicz, and the ISOLDECollaboration, Eur. Phys. A 10, 139 (2001).

[50] G. J. Farooq-Smith, Ph.D. thesis, KU Leuven, 2019, https://cds. cern.ch/record/2698502.

[51] M. Seliverstov, A. Barzakh, R. Ahmed, K. Chrysalidis, T. D. Goodacre, D. Fedorov, V. Fedoseev, C. Granados, B. Marsh, P. Molkanov, V. Panteleev, R. E. Rossel, S. Rothe, and S. Wilkins, Hyperfine Interact. 241, 40 (2020).

[52] A. Bohr and V. F. Wiesskopf, Phys. Rev. 77, 94 (1950).

[53] P. A. V. Bout, V. J. Ehlers, W. A. Nierenberg, and H. A. Shugart, Phys. Rev. 158, 1078 (1967).

[54] H. Dahmen and S. Penselin, Z. Phys. 200, 456 (1967).

[55] G. Passler, J. Rikovska, E. Arnold, H.-J. Kluge, L. Monz, R. Neugart, H. Ravn, and K. Wendt, Nucl. Phys. A 580, 173 (1994).

[56] F. D. Feiock and W. R. Johnson, Phys. Rev. 187, 39 (1969).

[57] A. Andreyev and A. Barzakh, CERN Document Server, Report No. CERN-INTC-2013-002 (CERN, Geneva, 2013), http://cds. cern.ch/record/1551259? ln=en.

[58] C. R. Bingham, M. B. Kassim, M. Zhang, Y. A. Akovali, K. S. Toth, W. D. Hamilton, H. K. Carter, J. Kormicki, J. von Schwarzenberg, and M. M. Jarrio, Phys. Rev. C 51, 125 (1995).
[59] P. G. Varmette, D. T. Shidot, W. C. Ma, A. V. Ramayya, R. V. F. Janssens, C. N. Davids, J. H. Hamilton, I. Ahmad, H. Amro, B. R. S. Babu, B. Back, K. S. Bindra, D. J. Blumenthal, L. T. Brown, M. P. Carpenter, W. L. Croft, B. Crowell, S. M. Fischer, U. Garg, R. G. Henry, T. Ishii, T. L. Khoo, J. Kormicki, T. Lauritsen, C. J. Lister, D. Nisius, H. Penttila, R. B. Piercey, J. A. Winger, S. J. Zhu, and P. B. Semmes, Phys. Lett. B 410, 103 (1997).

[60] C. Guo-Jie, S. Hui-Chao, and L. Yu-Xin, Chin. Phys. Lett. 22, 50 (2004).

[61] C. J. Gallagher and S. A. Moskowski, Phys. Rev. 111, 1282 (1958).

[62] C. Ekström, G. Wannberg, and Y. S. Shishodia, Hyperfine Interact. 1, 437 (1975).

[63] I.-L. Lamm, Nucl. Phys. A 125, 504 (1969).

[64] R. Bengtsson, J. Dudek, W. Nazarewicz, and P. Olanders, Phys. Scr. 39, 196 (1989).

[65] G. D. Dracoulis, B. Fabricius, R. A. Bark, A. E. Stuchbery, D. G. Popescu, and T. Kibedi, Nucl. Phys. A 510, 533 (1990).

[66] N. J. Stone, At. Data Nucl. Data Tables 90, 75 (2005).

[67] C. V. Beveren, A. N. Andreyev, A. E. Barzakh, T. E. Cocolios, R. P. de Groote, D. Fedorov, V. N. Fedosseev, R. Ferrer, L. Ghys, M. Huyse, U. Köster, J. Lane, V. Liberati, K. M. Lynch, B. A. Marsh, P. L. Molkanov, T. J. Procter, E. Rapisarda, K. Sandhu, M. D. Seliverstov, P. V. Duppen, M. Venhart, and M. Veselský, J. Phys. G: Nucl. Part. Phys. 43, 025102 (2016).

[68] J. O. Rasmussen, Phys. Rev. 113, 1593 (1959).

Correction: An affiliation for the eighth author was improperly presented as a byline footnote and has been moved into the list of affiliations. Subsequent affiliations and footnotes have been reordered. 